\title{
Neurexin and Neuroligins Maintain the Balance of Ghost and Satellite Boutons at the Drosophila Neuromuscular Junction
}

OPEN ACCESS

Edited by:

Fiorenzo Conti,

Marche Polytechnic University, Italy

Reviewed by:

Joachim H. R. Lübke, Julich Research Centre, Helmholtz Association of German Research

Centers (HZ), Germany

Manzoor A. Bhat,

The University of Texas Health

Science Center at San Antonio,

United States

Marta Pallotto

National Institutes of Health (NIH),

United States

*Correspondence:

Gan Guangming

ganguangm@seu.edu.cn

Received: 12 December 2019 Accepted: 26 March 2020

Published: 09 June 2020

Citation:

Guangming G, Junhua $G$, Chenchen $Z$, Yang $M$ and Wei $X$ (2020) Neurexin and Neuroligins

Maintain the Balance of Ghost and Satellite Boutons at the Drosophila Neuromuscular Junction. Front. Neuroanat. 14:19. doi: 10.3389/fnana.2020.00019

\author{
Gan Guangming ${ }^{1,2 *}$, Geng Junhua ${ }^{2}$, Zhang Chenchen ${ }^{1,2}$, Mou Yang ${ }^{1}$ and Xie Wei, ${ }^{2,3}$ \\ ${ }^{1}$ School of Medicine, Southeast University, Nanjing, China, ${ }^{2}$ The Key Laboratory of Developmental Genes and Human \\ Disease, Institute of Life Sciences, Southeast University, Nanjing, China, ${ }^{3}$ Institute of Life Sciences, The Collaborative \\ Innovation Center for Brain Science, Southeast University, Nanjing, China
}

Neurexins and neuroligins are common synaptic adhesion molecules that are associated with autism and interact with each other in the synaptic cleft. The Drosophila neuromuscular junction (NMJ) bouton is a well-known model system in neuroscience, and ghost and satellite boutons, respectively, indicate the poor development and overgrowth of the NMJ boutons. However, the Drosophila neurexin (DNrx) and Drosophila neuroligins (DNlgs) are mainly observed in type lb boutons, indicating the ultrastructural and developmental phenotypes of the Drosophila NMJ. Here, we identified the ultrastructural and developmental features of ghost and satellite boutons by utilizing dneurexin ( $d n r x)$ and dneuroligins (dnlgs) fly mutants and other associated fly strains. Ghost boutons contain synaptic vesicles with multiple diameters but very rarely contain T-bar structures and swollen or thin subsynaptic reticulum (SSR) membranes. The muscle cell membrane is invaginated at different sites, stretches to the ghost bouton from different directions, forms several layers that enwrap the ghost bouton, and then branches into the complex SSR. Satellite boutons share a common SSR membrane and present either a typical profile in which a main bouton is encircled by small boutons or two atypical profiles in which the small boutons are grouped together or distributed in beads without a main bouton. Electron and confocal microscopy data showed that $d n r x, d n \lg 1, d n \lg 2, d n \lg 3$, and $d n \lg 4$ mutations led to ghost boutons; the overexpression of $d n r x, d n \lg 1, d n \lg 2, d n \lg 3$, and dnlg4 led to satellite boutons; and the dnlg2; $d n \lg 3$ double mutation also led to satellite boutons. These results suggested that DNrx and DNlgs jointly maintain the development and function of NMJ boutons by regulating the balance of ghost and satellite boutons in Drosophila.

Keywords: neurexin, neuroligins, Drosophila, neuromuscular junction, ghost bouton, satellite boutons

\section{INTRODUCTION}

Neurexins and neuroligins are pre- and postsynaptic adhesion molecules, respectively, that are associated with autism. Caki interacts with neurexin before synapse formation and is involved in synaptic development (Sun et al., 2009), and neurexins and neuroligins interact with each other in the synaptic cleft. Moreover, $d n r x$ (Li et al., 2007; Rui et al., 2017), dnlg1 (Banovic et al., 2010), dnlg2 
(Sun et al., 2009, 2011), dnlg3 (Xing et al., 2014), and $d n \lg 4$ (Li et al., 2013; Zhang et al., 2017) are reportedly involved in synapse formation and synaptic transmission in Drosophila neuromuscular junction (NMJ) type I boutons. Furthermore, $d n r x$ (Larkin et al., 2015; Tong et al., 2016) and $d n l g 4$ (Li et al., 2013) were shown to disrupt Drosophila sleep patterns. Research based on the Drosophila larval NMJ model showed that the number of synaptic boutons was reduced in $d n r x$ ( $\mathrm{Li}$ et al., 2007; Rui et al., 2017), $d n \lg 1$ (Banovic et al., 2010), $d n \lg 2$ (Sun et al., 2009, 2011), and $d n \lg 4$ (Li et al., 2013; Zhang et al., 2017) mutants, and this phenotype could be rescued in all mutants by a gene overexpression system. The number of synaptic boutons increased and could also be rescued in the $d n \lg 3$ mutant (Xing et al., 2014). Furthermore, the number of synaptic boutons increased in overexpression strains of $d n r x$ (Li et al., 2007; Rui et al., 2017) and $d n \lg 2$ (Sun et al., 2009, 2011) and decreased in the $d n l g 3$ (Xing et al., 2014) overexpression strain. These results showed that $d n r x, d n \lg 1, d n \lg 2, d n \lg 3$, and $d n \lg 4$ together maintain the number of type I boutons.

The Drosophila larval NMJ is a well-known model system for studying synaptic development, signal transmission, and neurological disease and is classified by three distinct types of synaptic boutons (types I, II, and III) according to size, subsynaptic reticulum (SSR) characteristics, neurotransmitter identity, and synaptic vesicle composition in wild-type organisms (Atwood et al., 1993; Jia et al., 1993; Monastirioti et al., 1995). Type I boutons, the main type in the sixth/seventh muscles, have been studied in research on gene functions and neurological disorders, such as spinocerebellar ataxia (Tsuda et al., 2005), amyotrophic lateral sclerosis (Chai et al., 2008; Tsuda et al., 2008), spinal muscular atrophy (Sen et al., 2011), and Alzheimer's disease (Zempel and Mandelkow, 2015). In type I boutons, abnormal morphological phenotypes are mainly characterized by two manifestations on confocal and transmission electron microscopy (TEM). The first phenotype constitutes abnormal ultrastructural characteristics, such as abnormalities in the SSR (Banerjee et al., 2017), synapse and T-bar structures (Xing et al., 2014), and synaptic vesicles (Xing et al., 2014). The other phenotype is the appearance of two types of abnormal boutons, namely, ghost boutons and satellite boutons, on confocal microscopy.

On confocal microscopy, ghost boutons contain synaptic vesicles but no active zones. These structures express a neuronal membrane marker recognized by the anti-HRP antibody (Sutcliffe et al., 2013) but lack the postsynaptic Discs-large (Dlg) protein and GluRs (Ataman et al., 2006; Menon et al., 2013; Sutcliffe et al., 2013; Piccioli and Littleton, 2014). Ghost boutons are associated with poor synaptic development (Sutcliffe et al., 2013). Satellite boutons are constituted by several small boutons that bud from a parent bouton present in a branch of the terminal arbor (Lee and $\mathrm{Wu}, 2010$ ), however, in wild-type larval NMJs, a branching parent bouton normally has at most two new branches (Miller et al., 2012). Satellite boutons have also been shown to bud from an axonal segment that connects two adjacent boutons (Torroja et al., 1999). Satellite boutons contain the presynaptic proteins Synapsin and Brp, and the postsynaptic proteins Dlg and GluRs are more common in mutants that display NMJ bouton overgrowth than in the wild type (Dickman et al., 2006). Satellite boutons are correlated with genes associated with human mental illnesses (Endris et al., 2002; Fuentes-Medel et al., 2009; Li et al., 2016).

Reports of ghost and satellite boutons are principally based on laser scanning confocal microscopy, which has low resolution, and only limited TEM ultrastructural data are available. Therefore, the ultrastructural phenotypes of the common synaptic adhesion molecules Drosophila neurexin (DNrx) and Drosophila neuroligins (DNlgs) are mainly determined from type Ib boutons. Because NMJ boutons are fairly diffuse in muscles and because the muscles in Drosophila larvae are often curled, researchers must prepare many slices to obtain sufficient NMJ bouton samples. In this study, we prepared a wide range of samples and analyzed the morphological properties and development of ghost and satellite boutons in Drosophila larvae with caki, $d n r x$, and $d n l g s$ mutants, the wild-type strain and a dbrat mutant with typical satellite boutons on confocal microscopy (Shi et al., 2013). The results of this study suggested that DNrx and DNlgs jointly maintain the balance of ghost and satellite boutons in the Drosophila NMJ.

\section{MATERIALS AND METHODS}

\section{Drosophila Stocks}

The $w^{1118}$ strain was used as the wild-type control in this study. All stocks were cultured in standard medium at $25^{\circ} \mathrm{C}$. Fly stocks Df(3R) X-313 (hereafter called caki ${ }^{313}$ ) and Df(3R)X-307 (hereafter called caki ${ }^{307}$ ) were obtained from the Bloomington Stock Center at Indiana University (Bloomington, IN, United States), and both strains were shown to carry a recessive lethal partial deletion of the caki gene (Sun et al., 2009). The $d b r a t^{11}$ and $d b r a t^{192}$ strains were obtained from Dr. Zhang and were previously shown to carry a recessive lethal partial deletion of the dbrat gene (Shi et al., 2013). The following fly mutants were used: $d n r x^{273}$ (Li et al., 2007), $d n \lg 1^{\text {ex1.9 }}$ and $d n \lg 1^{\text {ex2.3 }}$ (Banovic et al., 2010), $d n \lg 2^{K O 70}$ and $d n \lg 3^{K O 127}$ (Xing et al., 2018), and $d n \lg 4^{\mathrm{KO} 10}$ (Zhang et al., 2017). The following $w^{1118}$ overexpression strains were used: $24 B$-Gal4;UASdnlg1, 24B-Gal4;UAS-dnlg2, MHC-Gal4;UAS-dnlg3 (Xing et al., 2014), and Ok6-Gal4;UAS-dnlg4 (Zhang et al., 2017), as well as $d n \lg 2^{\mathrm{KO} 70} ; \operatorname{dnlg} 3^{\mathrm{KO} 127}$, Ok6-Gal4;UAS-dnrx, and MHCGal4;UAS-dnlg1, which were generated in our laboratory. The rescue strains were 24B-Gal4;UAS-dnlg2 (Sun et al., 2011) and Elav-Gal4;UAS-dnlg3 (Xing et al., 2014).

\section{TEM of Larval NMJ Boutons}

Dissection and fixation were based on standard procedures (Xing et al., 2018). In brief, the wandering late third-instar larvae were dissected with standard techniques in Jan solution (128 mM NaCl, $2 \mathrm{mM} \mathrm{KCl,} 4 \mathrm{mM} \mathrm{MgCl}_{2}, 35 \mathrm{mM}$ sucrose, $5 \mathrm{mM}$ HEPES, $\mathrm{pH}$ 7.4) and fixed at $4^{\circ} \mathrm{C}$ overnight in a mixture of $2 \%$ glutaraldehyde and $2 \%$ formaldehyde in $0.1 \mathrm{M}$ sodium cacodylate buffer ( $\mathrm{pH}$ 7.4), followed by several rinses with cacodylate buffer. The samples were then postfixed for $2 \mathrm{~h}$ with $1 \% \mathrm{OsO}_{4}$ in cacodylate buffer and rinsed twice with distilled 

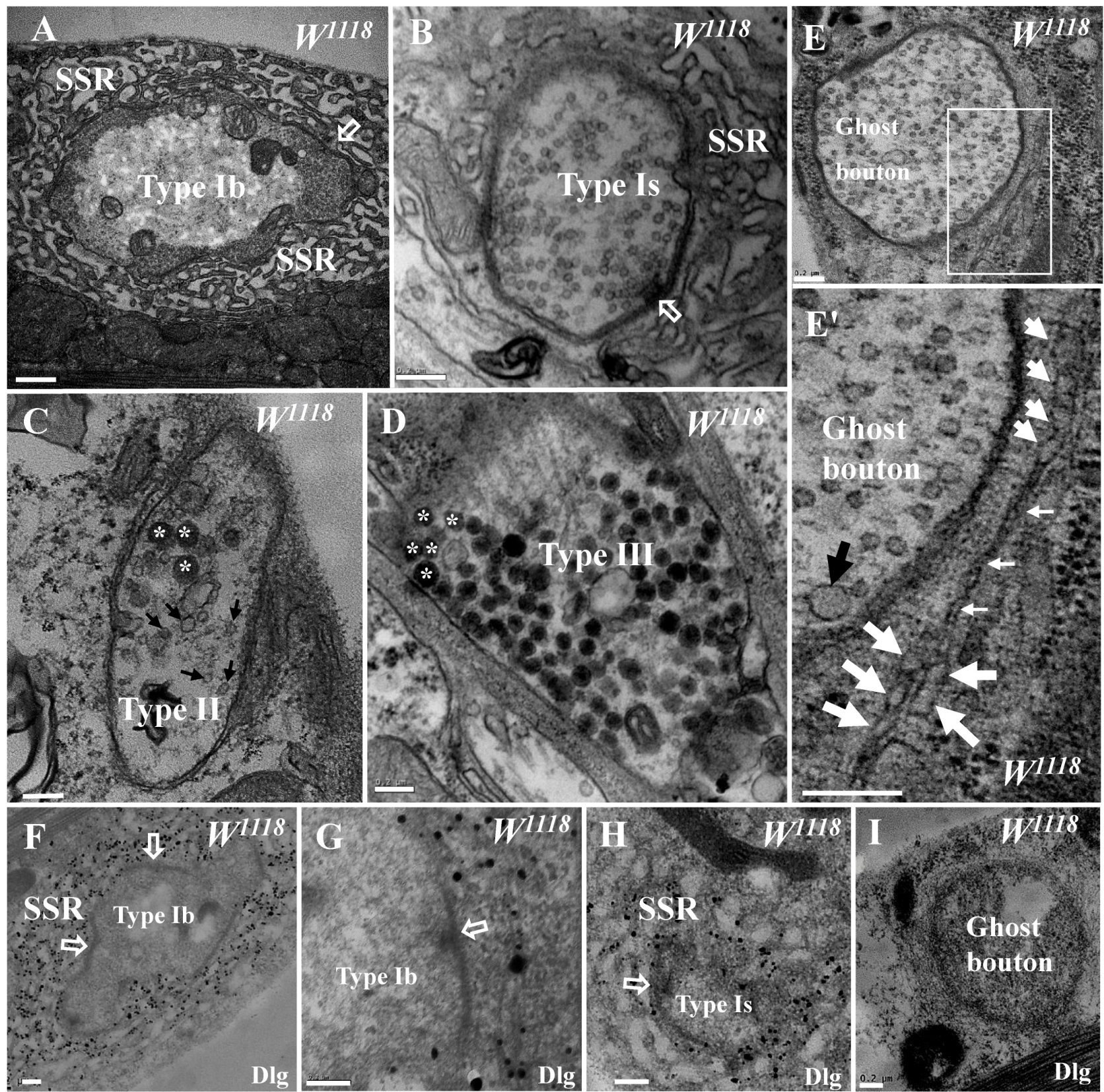

FIGURE 1 | General characteristics of type I, type II, type III, and ghost boutons in wild-type samples. A type Ib bouton with a thick subsynaptic reticulum (SSR) and T-bar (A) and a type Is bouton with a less developed SSR and T-bar (B). A type II bouton contains dense core vesicles and clear vesicles without an SSR (C), and a type III bouton contains dense core vesicles without an SSR (D). Ghost boutons have no T-bar structures or SSR membranes (E) with large synaptic vesicles (E') and rare swollen SSR membranes ( $\left.\mathbf{E}^{\prime}\right)$. Dlg signals (black dots) were dense in the SSR membranes of type lb boutons (F,G) and sparse in type lb boutons

(H) but not in ghost boutons (I) or in the first muscle cell membrane of type lb boutons (G). Hollow arrows show T-bars and synapses, large black arrows show large clear vesicles, small black arrows show small clear vesicles, and asterisks indicate dense core vesicles. Thick white arrows show swollen SSR membranes, and thin white arrows show thin SSR membranes. (E') is an enlargement of the white box in (E). Scale bars: (A), $500 \mathrm{~nm}$; (B-I), $200 \mathrm{~nm}$.

water. The preparations were stained for $2 \mathrm{~h}$ with $2 \%$ saturated uranyl acetate in distilled water and rinsed twice with distilled water. The specimens were dehydrated in an ethanol series, passed through propylene oxide two times, and embedded into a sheet in Epon812 (SPI Science). A total of 80 microns was serially sectioned using a diamond knife on a Leica UC7 ultrathin microtome at the sixth/seventh muscles of the $\mathrm{A}_{3}$ or $\mathrm{A}_{2}$ segment in one animal; each slice was $90 \mathrm{~nm}$ thick, 30-40 slices were gathered into a group and attached to a grid, and approximately 30 grids were used. The grids were poststained with $2 \%$ saturated uranyl acetate in $50 \%$ ethanol and $1 \%$ lead citrate $(\mathrm{pH} 12)$ and examined under a transmission electron microscope (Hitachi 
TABLE 1 | Ghost boutons and satellite boutons analysis in TEM.

\begin{tabular}{|c|c|c|c|c|c|c|c|c|c|c|c|}
\hline Genotype & WT & caki & dnrx & $d n l g l$ & dnlg2 & dnlg3 & $d n \lg 4$ & $O E-d n / g 2$ & $O E-d n l g 3$ & $d n \lg 2 ; d n / g 3$ & dbrat \\
\hline Ghost boutons per $6 / 7$ muscle & $3 / 15$ & $0 / 3$ & $5 / 2$ & $0 / 3$ & $2 / 4$ & $0 / 4$ & $9 / 3$ & $0 / 2$ & $0 / 3$ & $0 / 2$ & $0 / 3$ \\
\hline Ghost boutons (SEM) & $0.2 \pm 0.11$ & 0 & $1.3 \pm 0.33$ & 0 & $1 \pm 0.78$ & 0 & $3 \pm 0.58$ & 0 & 0 & 0 & 0 \\
\hline Ghost boutons $P$-value & & & 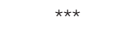 & & * & & $* \star \star$ & & & & \\
\hline Satellite boutons per $6 / 7$ muscle & $0 / 15$ & $0 / 3$ & $0 / 3$ & $0 / 3$ & $0 / 4$ & $0 / 4$ & $0 / 3$ & $0 / 3$ & $19 / 2$ & $15 / 2$ & $12 / 3$ \\
\hline
\end{tabular}

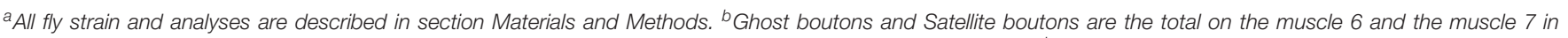

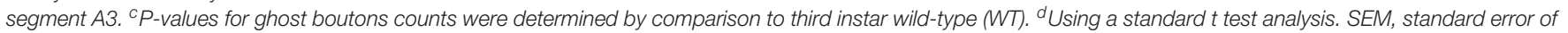
the mean. ${ }^{*} p<0.05 ;{ }^{* * *} p<0.001$.

H-7650). More than 20 wild-type animals were analyzed, and 3 animals were analyzed for the other strains.

\section{The Procedure for Pre-embedding Immunogold Electron Microscopy}

Pre-embedding immunogold electron microscopy was performed based on standard procedures (Gan and Zhang, 2018). In brief, wandering late third-instar larvae were dissected and fixed in a mixed agent ( $4 \%$ formaldehyde, $0.5 \%$ glutaraldehyde, and $10 \%$ saturated picric acid in $0.1 \mathrm{M}$ sodium cacodylate buffer, $\mathrm{pH}$ 7.4) for $4 \mathrm{~h}$. Then, the samples were washed with $0.1 \mathrm{M}$ sodium cacodylate buffer diluted with $1 \%$ saponin for $1 \mathrm{~h}$, preincubated in $0.1 \%$ BSA with $0.1 \%$ saponin for $1 \mathrm{~h}$, and incubated with a mouse anti-Dlg primary antibody (DSHB) for $24 \mathrm{~h}$ at $4^{\circ} \mathrm{C}$. After four rinses with $0.1 \%$ Tween-20, the samples were preincubated with $0.1 \%$ gelatin, $0.5 \%$ BSA and $0.1 \%$ saponin in $0.1 \mathrm{M}$ PBS for $1 \mathrm{~h}$, incubated with a $1.4 \mathrm{~nm}$ ultrasmall gold-conjugated secondary antibody (goat anti-mouse IgG secondary antibody, Nanoprobes, \#2001, 1:50) for $1 \mathrm{~h}$ at $4^{\circ} \mathrm{C}$, and rinsed four times with $0.1 \%$ Tween-20 in $0.1 \mathrm{M}$ PBS. The samples were then postfixed in $2.0 \%$ glutaraldehyde in PBS for $30 \mathrm{~min}$ and rinsed several times with distilled water. Silver enhancement (HQS kit; Nanoprobes, \#2012) was performed in the dark at $4^{\circ} \mathrm{C}$ for $25 \mathrm{~min}$, followed by rinsing with distilled water. After rinsing with PBS for $10 \mathrm{~min}$, the samples were either osmicated in $0.1 \mathrm{M}$ sodium cacodylate for $1 \mathrm{~h}$ or not. All samples were washed three times with distilled water and then stained with $2 \%$ aqueous uranyl acetate for $2 \mathrm{~h}$ at $4^{\circ} \mathrm{C}$. Subsequent epoxy resin embedding, trimming, and thin sectioning were performed as described above for the NMJ boutons at the sixth/seventh muscles in the $\mathrm{A}_{3}$ or $\mathrm{A}_{2}$ segment.

\section{Immunochemistry}

Immunostaining of the larval samples was performed as described previously (Xing et al., 2014). Briefly, the larval samples were fixed for $40 \mathrm{~min}$ in paraformaldehyde, washed four times with PBS and $0.3 \%$ PBST (0.3\% Triton X-100 in PBS), blocked in $1 \%$ bovine serum albumin for $1 \mathrm{~h}$, incubated with antiHrp (Jackson ImmunoResearch, West Grove, PA, United States) or anti-Dlg (4F3; 1:50; DSHB) at $4^{\circ} \mathrm{C}$ for $2 \mathrm{~h}$, and incubated with fluorophore-conjugated secondary antibodies (Invitrogen, 1:500) for $1 \mathrm{~h}$ at room temperature. The samples were washed extensively and mounted in VectaShield mounting medium
(Vector Laboratories). The images were collected using an Olympus FV3000 confocal microscope.

\section{Statistical Analysis}

The ghost and satellite boutons were counted at the sixth/seventh muscles of the A2-A3 segments from Drosophila larvae. As to each strain, at least eight segments were counted for confocal microscopy data, and at least three segments were counted for TEM data. The data were analyzed with GraphPad Prism 5 using unpaired, two-tailed $t$-tests.

\section{RESULTS}

\section{Identification of Ghost Boutons in Wild-Type Flies by TEM}

Drosophila larval NMJs were classified as type I, type II, and type III boutons according to their size, SSR characteristics, and synaptic vesicle composition under TEM (Atwood et al., 1993; Jia et al., 1993). The type Ib bouton contained a thick SSR with a large size (Figure 1A), and the type Is bouton had a less developed SSR with a small size (Figure 1B) in the wild-type flies. Both type II and type III boutons lacked the distinctive SSR, type II boutons contained both dense core vesicles and small clear vesicles (Figure 1C), and type III boutons mainly contained dense core vesicles (Figure 1D).

Ghost boutons are extremely rarely observed under confocal microscopy, and the current literature has not described these structures in wild-type Drosophila under TEM. We found ghost boutons in wild-type Drosophila in a wide range of serial sections from more than 20 animals. The ghost boutons had no synapses, T-bar structures, or SSR membranes (Figure 1E) but had synaptic vesicles with various diameters (Figure $\mathbf{1 E}^{\prime}$ ). There were two layers of paired membranes in the ghost boutons: the inner membrane was from the axon terminal, and the other membrane was from the muscle cell membrane. The ghost boutons were extremely rarely observed with TEM, and we observed only one ghost bouton between the sixth and seventh muscles in more than five animals. Occasionally, two SSR membrane layers were observed to stretch along a ghost bouton, and the SSR membranes did not branch (Figure 1E' $\mathbf{E}^{\prime}$ ). A considerable fraction of the SSR membranes were swollen (Figure 1E' $\mathbf{E}^{\prime}$ ).

$\mathrm{Dlg}$ is widely used as a conventional molecular marker of postsynaptic SSR membranes in Drosophila NMJ boutons under confocal microscopy. Using a pre-embedding immunogold 

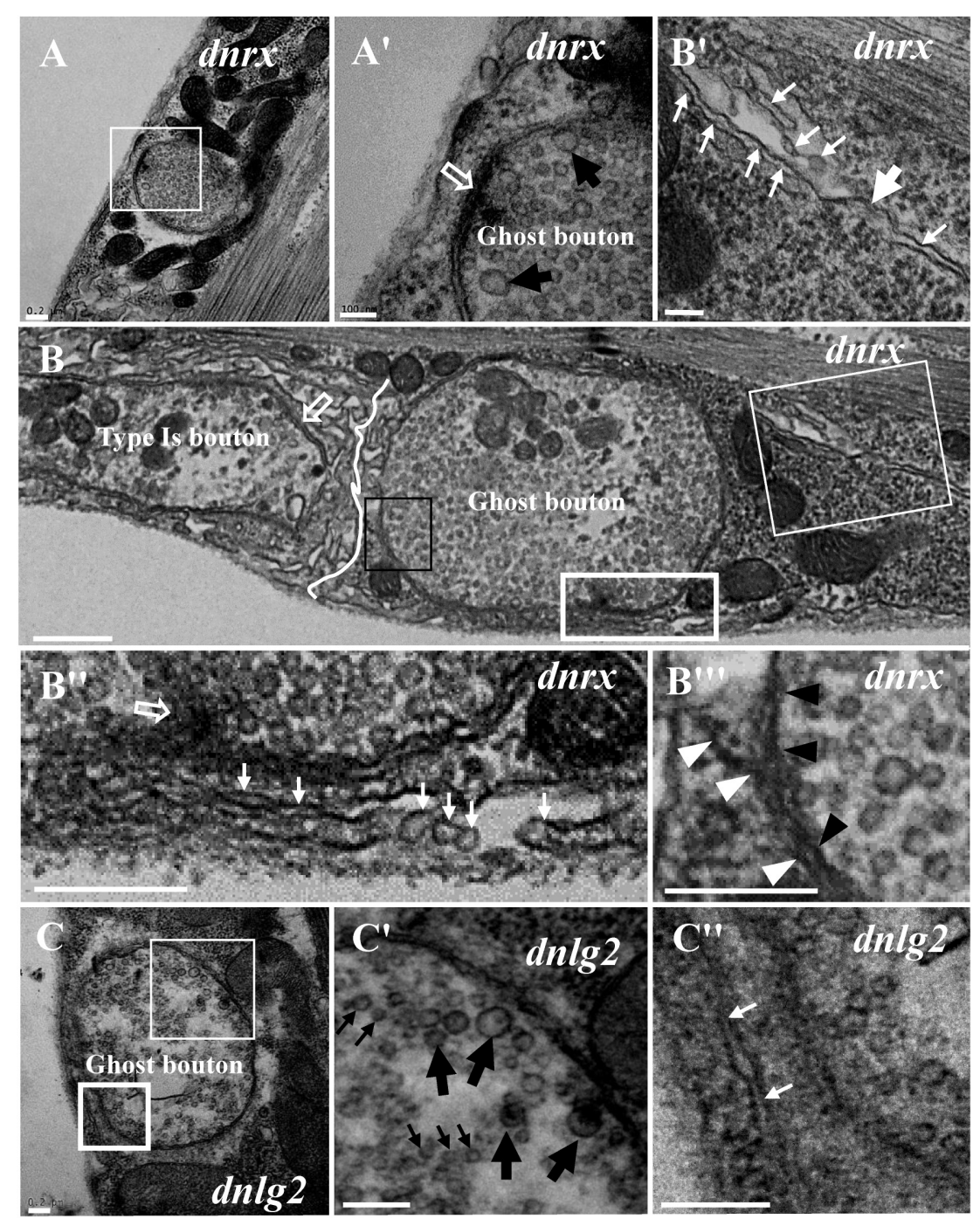

FIGURE 2 | Ultrastructure of the ghost boutons in the dnrx and dnlg2 mutants. In the dnrx mutant, ghost boutons have large synaptic vesicles, a synapse and T-bar structure, and no SSR membrane (A, $\left.\mathbf{A}^{\prime}\right)$. A developing ghost bouton has an obvious boundary with a nearby type ls bouton at one end (B) and a T-bar (B, $\left.\mathbf{B}^{\prime \prime}\right)$ and a sparse, thin SSR membrane at the other end $\left(\mathbf{B}, \mathbf{B}^{\prime}, \mathbf{B}^{\prime \prime}\right)$. The first muscle cell membrane and the axonal membrane are separated in the ghost bouton $\left(\mathbf{B}, \mathbf{B}^{\prime \prime \prime}\right)$. In the dnlg2 mutant, ghost boutons have large synaptic vesicles (C, $\left.\mathbf{C}^{\prime}\right)$ and only one single flat SSR membrane (C, $\left.\mathbf{C}^{\prime \prime}\right)$. Hollow arrows show T-bars or synapses, large black arrows show large clear vesicles, and small black arrows show small clear vesicles. Thick white arrows show swollen SSR membranes, and thin white arrows show thin SSR membranes. The paired axonal membrane (black wedges) and muscle cell membrane (white wedges) were separated in ghost boutons. $\left(\mathbf{A}^{\prime}, \mathbf{B}^{\prime}, \mathbf{C}^{\prime}\right)$ Are the enlarged images of the thin white boxes in $\mathbf{( A - C )}$, respectively. $\left(\mathbf{B}^{\prime \prime}, \mathbf{C}^{\prime \prime}\right)$ Are the enlarged images of the thick white boxes in $(\mathbf{B}, \mathbf{C})$, respectively. $\left(\mathbf{B}^{\prime \prime \prime}\right)$ Is an

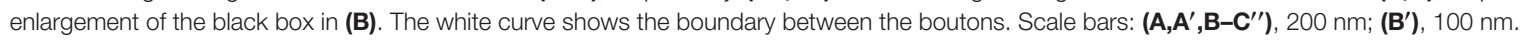

electron microscopy method, we observed that the Dlg protein was widely distributed in the SSR membranes of type Ib boutons (Figures 1F,G) and type Ib boutons (Figure 1H; Gan and Zhang, 2018) but was not present in the ghost boutons (Figure 1I).

\section{The dnrx, dnlg2, and dnlg4 Mutants Had Increased Levels of Ghost Boutons Under TEM}

We frequently found ghost boutons in the $d n r x$, dnlg2, and dnlg4 mutants (Table 1). We observed the synapse and T-bar structure in a ghost bouton without an SSR membrane in the dnrx mutant (Figures 2A, $\mathbf{A}^{\prime}$ ). A long, sparse SSR membrane stretched from the upper (Figures $\mathbf{2} \mathbf{B}, \mathbf{B}^{\prime}$ ) and lower directions (Figures 2B, $\mathbf{B}^{\prime \prime \prime}$ ), not around the partially naked ghost bouton but in the opposite region of the same ghost bouton that was adjacent to a type Is bouton (Figure 2B). The SSR membranes had an obvious boundary between the ghost bouton and type Is bouton (Figure 2B). The two-layer membranes of the developing ghost boutons, including the inner axon terminal membrane and the outer muscle cell membrane, were separated, and the outer layer participated in the formation of the SSR (Figures 2B, $\mathbf{B}^{\prime \prime \prime}$ ). Moreover, the ghost bouton contained a synapse and T-bar structure with sparse SSR membranes 

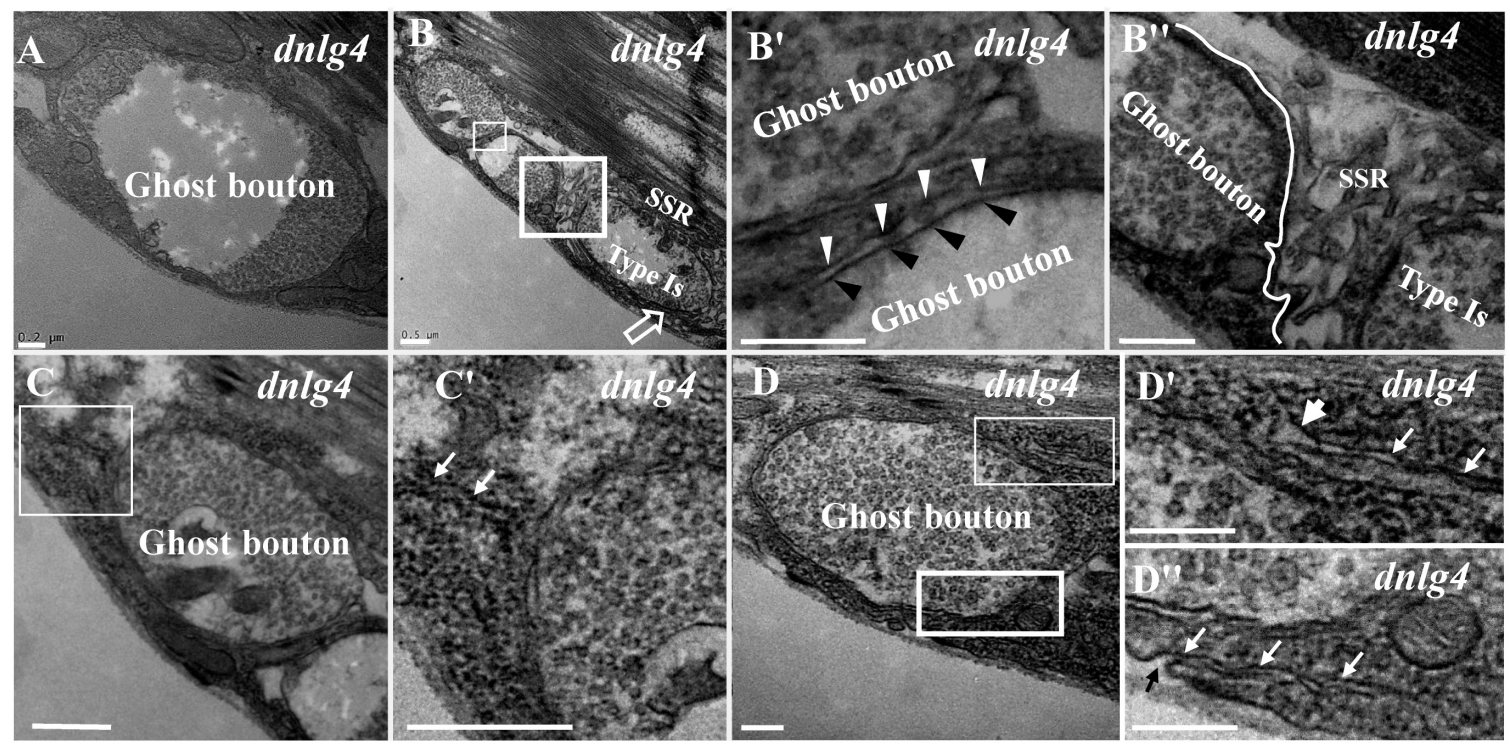

FIGURE 3 | Ultrastructure of the ghost boutons in the dnlg4 mutant. Ghost boutons have no synapses, T-bar structures, or SSR in the dnlg4 mutant (A). Beaded ghost boutons in the dnlg4 mutant (B). No SSR membranes are observable between the ghost boutons (B, $\left.\mathbf{B}^{\prime}\right)$. The ghost bouton does not share SSR membranes with the adjacent type Is boutons (B, $\mathbf{B}^{\prime \prime}$ ). Sparse thin SSR membranes extend to ghost boutons in the dnlg4 mutant (C,C'). Thin SSR membranes around the ghost bouton from the upper direction (D, $\left.\mathbf{D}^{\prime}\right)$ and downward direction (D, $\left.\mathbf{D}^{\prime \prime}\right)$ in the dnlg4 mutant. Hollow arrows show T-bars or synapses. Thick white arrows show swollen SSR membranes, and thin white arrows show thin SSR membranes. The axonal membrane (black wedges) is a paired muscle cell membrane (white

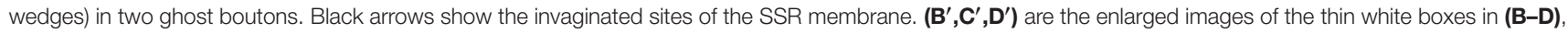

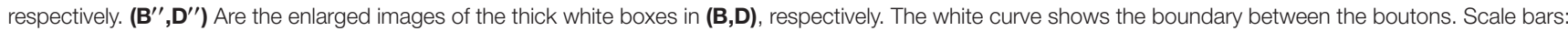
(A, $\left.\mathbf{B}^{\prime \prime}, \mathbf{D}-\mathbf{D}^{\prime \prime}\right), 200 \mathrm{~nm} ;\left(\mathbf{B}, \mathbf{B}^{\prime}, \mathbf{C}, \mathbf{C}^{\prime}\right), 500 \mathrm{~nm}$.

(Figures 2B, $\mathbf{B}^{\prime \prime}$ ), which showed that the ghost bouton was developing and forming the SSR.

Furthermore, we observed ghost boutons in the $d n \lg 2$ mutant (Figures $\mathbf{2 C}-\mathbf{C}^{\prime \prime}$ ). The ghost boutons had synaptic vesicles with different diameters (Figures $\mathbf{2 C}, \mathbf{C}^{\prime}$ ) and a single flat SSR membrane (Figures $\mathbf{2 C}, \mathbf{C}^{\prime \prime}$ ) or no SSR membrane.

Ghost boutons had synaptic vesicles with different diameters and no synapses, T-bar structures, or SSR membranes (Figure 3A) in the $d n \lg 4$ mutant; the ghost boutons formed bead shapes (Figures $\mathbf{3 B}, \mathbf{B}^{\prime}$ ) in some NMJ branches without SSR membranes or T-bar structures and resembled normal type Is boutons (Figures $\mathbf{3 B}, \mathbf{B}^{\prime \prime}$ ) with SSR membranes and T-bar structures. Notably, no SSR membrane was observed between the two ghost boutons, and there were two layers of paired membranes in each ghost bouton (Figures $\mathbf{3} \mathbf{B}, \mathbf{B}^{\prime}$ ). The inner membrane was from the axon terminal, and the other membrane was from the muscle cell membrane (Figure $3 \mathbf{B}^{\prime}$ ). Additionally, the ghost bouton did not share the SSR membrane with the adjacent type Is bouton (Figures $3 \mathbf{B}, \mathbf{B}^{\prime \prime}$ ). The extremely sparse SSR membranes were near (Figures $\mathbf{3 C}, \mathbf{C}^{\prime}$ ) or stretched toward and touching (Figure 3D) the ghost boutons from the upper (Figures 3D,3D') and lower directions (Figures 3D,3D') but were not wrapped around the ghost bouton. Furthermore, few SSR membranes were swollen (Figure $3 \mathrm{D}^{\prime}$ ), and most SSR membranes were thin (Figures $3 \mathrm{D}^{\prime}, \mathbf{3} \mathrm{D}^{\prime \prime}$ ). The invaginated site was connected to a long thin SSR membrane (Figure 3D' ${ }^{\prime \prime}$ ).

Because the extremely sparse SSR membranes stretched toward and touched the ghost boutons from different directions
(Figures 3D-D ${ }^{\prime \prime}$ ), we speculated that different invaginated sites were present on the cell membrane near the NMJ bouton. We examined the cell membrane surfaces in the NMJ bouton sections and found that all type Is (Figures $\mathbf{4} \mathbf{A}-\mathbf{A}^{\prime \prime}$ ) and type Ib (Figures 4B, $\mathbf{B}^{\prime}$ ) boutons had several invaginated sites on the cell membrane despite that the invaginated entrance was clear and distinct (Figures $\mathbf{4} \mathbf{A}^{\prime}, \mathbf{A}^{\prime \prime}$ ), demonstrating that complex SSR membranes were invaginated by muscle cell membranes at multiple sites. Moreover, these invaginated SSR membranes were repeatedly branched (Figures $4 \mathbf{B}^{\prime}, \mathbf{B}^{\prime \prime}$ ) to form the complex SSR of NMJ boutons (Figures 4A,B). Fortunately, we observed an axon terminal that was enwrapped by several layers of SSR membranes in the $d n \lg 1$ mutant (Figures 4C,D). The three sites that adhered to the extracellular matrix were clear and distinct (Figures $4 D, D^{\prime}$ ) and showed several independent clear SSR membranes wrapped around the axon terminal (Figures $\mathbf{4} \mathbf{C}, \mathbf{C}^{\prime}, \mathbf{D}, \mathbf{D}^{\prime \prime}$ ). These data demonstrated the SSR formation process. Initially, the muscle cell membrane invaginates from different sites and extends to a ghost bouton. Then, the ghost bouton is enwrapped with several layers of membranes. Finally, these membranes repeatedly branch and form a complex SSR membrane.

\section{Identification of the Three Types of Satellite Boutons on TEM}

Because we previously demonstrated by confocal laser scanning microscopy that dbrat mutants had numerous satellite boutons (Shi et al., 2013), we used a dbrat mutant to identify the 


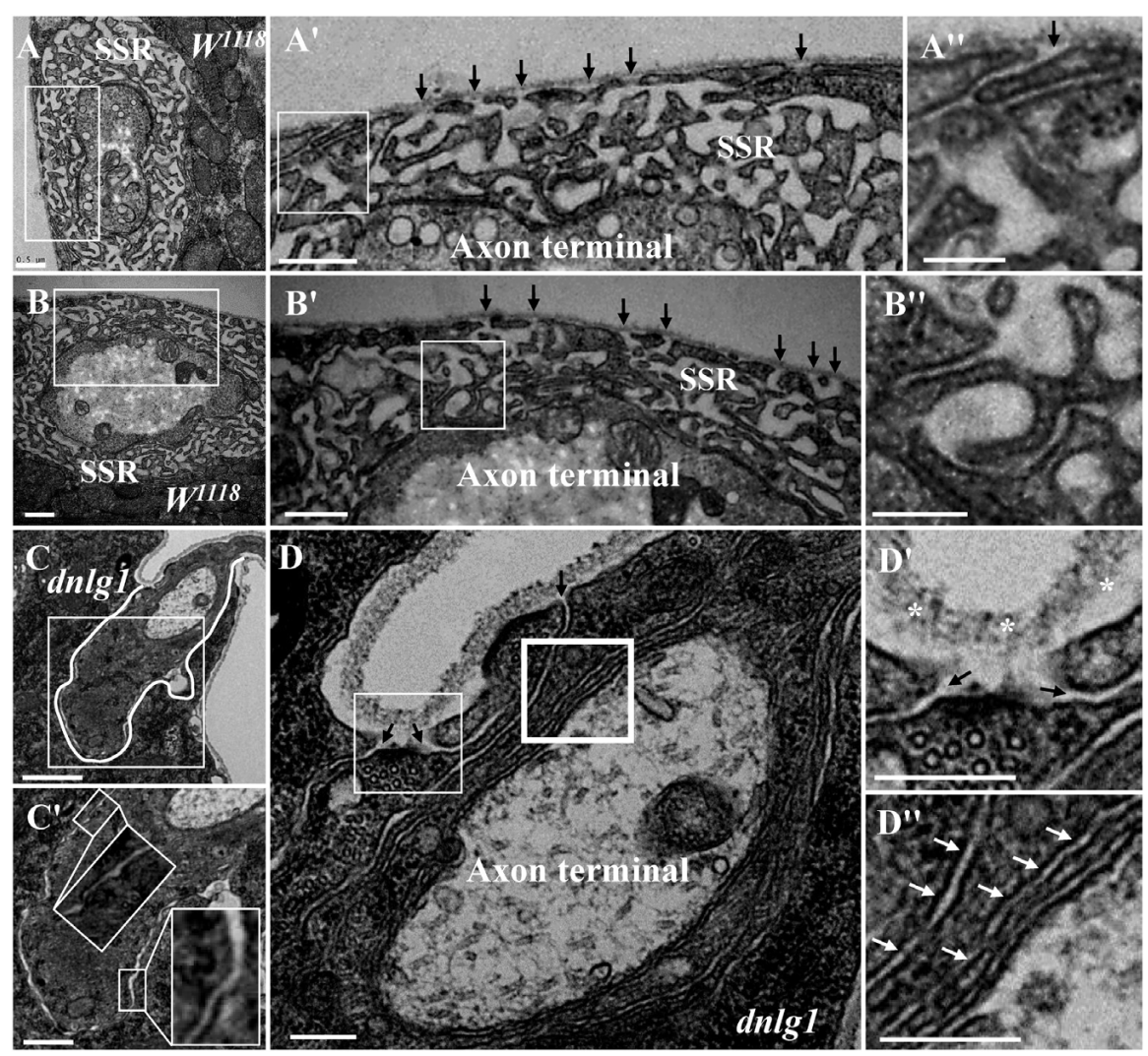

FIGURE 4 | Muscle cell membranes invaginate at multiple sites and form SSR membranes. Six invaginated sites exist outside of the type Is boutons (A,A') in the wild-type strain. An invaginated site with a long SSR membrane $\left(\mathbf{A}^{\prime \prime}\right)$. Seven invaginated sites exist outside of the type lb bouton (B, $\left.\mathbf{B}^{\prime}\right)$ in the wild-type strain. The

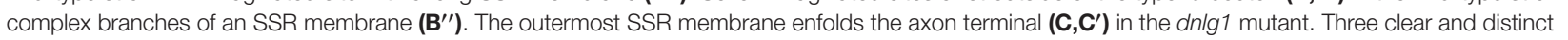

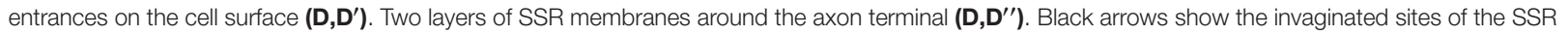

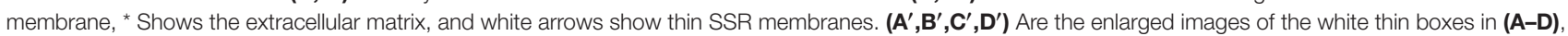

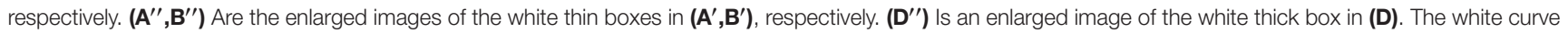
shows the boundary of the bouton. Scale bars, (A,A', $\left.\mathbf{B}, \mathbf{B}^{\prime}, \mathbf{C}^{\prime}\right), 500 \mathrm{~nm} ;\left(\mathbf{A}^{\prime \prime}, \mathbf{B}^{\prime \prime}, \mathbf{D}-\mathbf{D}^{\prime \prime}\right), 200 \mathrm{~nm} ;(\mathbf{C}), 1,000 \mathrm{~nm}$.

ultrastructural features of satellite boutons as a positive control. Under TEM, satellite boutons had typical and atypical forms. Typical satellite boutons consisted of a large main bouton and several small boutons observable in serial sections (Figures 5AD); the main bouton was in the center, and the small boutons, most less than $500 \mathrm{~nm}$ in diameter and appearing as several "satellites," were spread around the main bouton in the dbrat mutant (Figures 5B-D). The synaptic vesicles were distributed around the main bouton and were absent in the center of the main bouton (Figures $5 \mathbf{A}, \mathbf{B}$ ), similar to the normal type I bouton in wild-type flies (Figure 1A). The small satellite boutons were completely filled with synaptic vesicles (Figures $\mathbf{5 B}^{\prime}, \mathbf{B}^{\prime \prime}, \mathbf{D}^{\prime}$ ), unlike the main bouton (Figures $\mathbf{5} \mathbf{A}, \mathbf{B}$ ). Synapses and typical T-bar structures were observed in the main bouton (Figures $\mathbf{5 A}, \mathbf{A}^{\prime}$ ) and in the satellite boutons (Figures 5 $\mathbf{B}^{\prime}, \mathbf{B}^{\prime \prime}, \mathbf{D}^{\prime}$ ).

In contrast to the typical satellite boutons, two types of atypical satellite boutons were observed. In the first type of atypical satellite bouton, the central main bouton was absent or small, and several small boutons were grouped together (Figures 5E,F) with synapses (Figures $\mathbf{5 E}^{\prime}, \mathbf{F}^{\prime}$ ), as observed in the $d n \lg 2 ; \operatorname{dnlg} 3$ double mutant. In the other atypical satellite boutons, there was no central main bouton, and the small irregular boutons were arranged in a bead-like shape in three dbrat mutants (Figures $6 \mathrm{C}-\mathbf{E}^{\prime}$ ) and in the dnlg2; dnlg3 double mutant (Figure 6F), showing a common SSR membrane. In contrast, in wild-type flies, the adjacent type Ib boutons were large and markedly spherical or ellipsoidal, with clear boundaries comprising the SSR membranes (Figure 6A). The small beaded boutons were occasionally visible in an interconnected SSR in the wild-type strain (Figure 6B), and a relatively obvious boundary (Figure 6 $\mathbf{B}^{\prime}$ ) was observable between the bead boutons. The small beaded boutons could not be identified as satellite boutons (Figures 6G-K) without a common SSR membrane by TEM, and even the small NMJ boutons were distributed in beads in the caki mutant under confocal microscopy (Sun et al., 2009). Obvious differences in the boundaries and orientation between the different small-sized boutons in the caki mutant (Figures 6G-I) were observed by TEM. Satellite boutons could be observed in the Elav-Gal4;UAS-dnlg3 strain and in the dbrat mutant (Table 1), but there were still notable boundaries between the small NMJ boutons; thus, these boutons could not be 

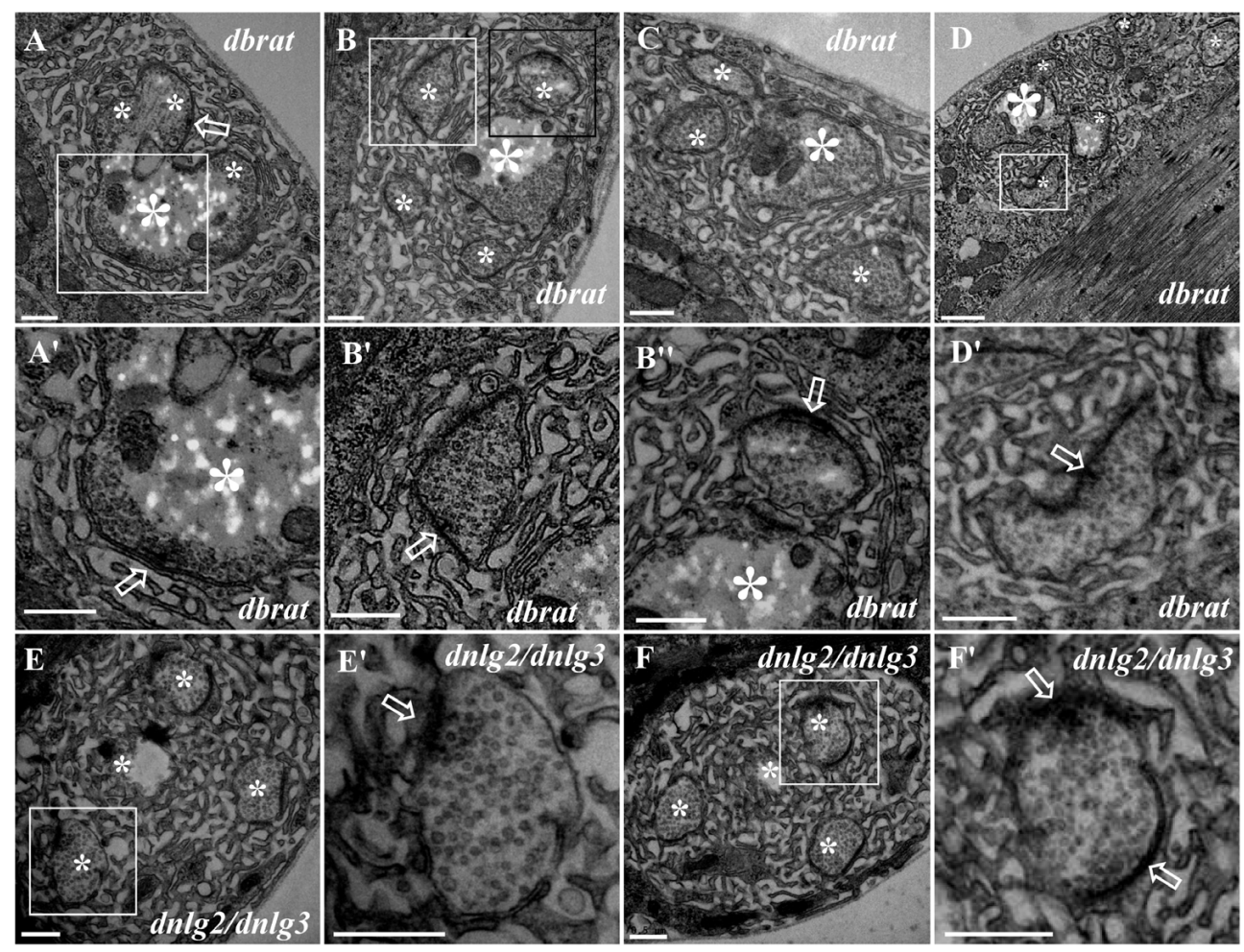

FIGURE 5 | Ultrastructure of the satellite boutons in the dbrat and dnlg2; dnlg3 mutants. Typical satellite boutons in the $d b r a t$ mutant contain a main bouton and

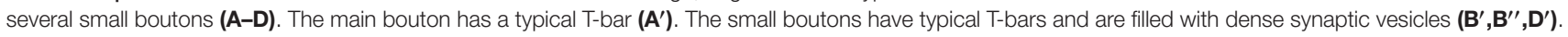
The atypical satellite boutons in the $d n l g 2 ; d n l g 3$ double mutant contain only four small boutons (E,F) that contain T-bars and are densely loaded with synaptic vesicles $\left(\mathbf{E}^{\prime}, \mathbf{F}^{\prime}\right)$. Hollow arrows show T-bars or synapses, large asterisks show the main boutons, and small asterisks show small boutons or branches.

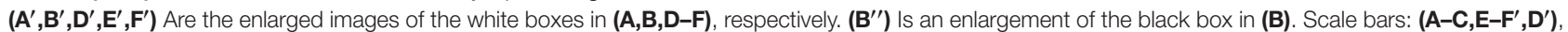
$500 \mathrm{~nm} ;$ (D), 1,000 nm.

considered satellite boutons because no common SSR was shared (Figures 8J, $\mathbf{J}^{\prime}, \mathbf{K}, \mathbf{K}^{\prime}$ ).

\section{DNIg2 and DNIg3 Synergistically Led to Satellite Boutons as Determined by TEM}

We did not observe satellite boutons in the $d n \lg 2$ (Figure 7A) mutants or rescued 24B-Gal4;UAS- $\ln \lg 3$ (Figure 7B) and $d n \lg 3$ (Figures 7C,C') mutants as reported by Sun et al. (2011) and Xing et al. (2018). However, we frequently observed typical satellite boutons in the $d n \lg 2 ; d n \lg 3$ double mutant (Figures 7D,E and Table 1). All main and small boutons shared a common SSR membrane that was fairly regular and layered, but the shape appeared to be extremely irregular in the large main boutons and relatively small main boutons, including cruciform boutons (Figure 7F), exogenous small boutons (Figure 7G), and small boutons repeatedly growing from a large NMJ bouton (Figure $\mathbf{7 H}$ ). Interestingly, more prominent typical satellite boutons were observed in the rescued MHC-Gal4;UAS-dnlg3 strain. We observed that five small boutons were distributed around the main bouton (Figures $7 \mathbf{I}, \mathbf{J}$ ) and the cruciform bouton (Figure $7 \mathbf{K}$ ). The large irregular boutons had a common SSR (Figures 7L,M), whereas the type Ib boutons were mostly spherical or ellipsoidal in wild-type flies (Figures 1A, 6A).

\section{The dnrx and dnlgs Jointly Maintained the Balance of Ghost and Satellite Boutons as Determined by Confocal Microscopy}

Our TEM data showed the ultrastructures of the ghost and satellite boutons in the $d n r x, d n \lg 2$, and dnlg4 mutants. The ghost and satellite boutons were observed under confocal microscopy with a large field and three-dimensional view, utilizing immunofluorescence labeling of Dlg, a marker for the postsynaptic SSR, and Hrp, a marker for the presynaptic membrane. To confirm whether the dnlg1 and $d n \lg 3$ mutants exhibited ghost boutons and whether the dnrx- and dnlgoverexpressing strains had satellite synapses, we analyzed the numbers of ghost and satellite boutons in the $d n r x$ and $d n l g$ mutants, $d n r x$ - and dnlg-overexpressing strains, and the associated double mutants by confocal microscopy (Figures 8, 9).

Consistent with the electron microscopy results, we rarely observed ghost boutons in the $W^{1118}$ strain $(0.063 \pm 0.043$, $N=32$ ) (Figures $\mathbf{8 A}-\mathbf{A}^{\prime \prime}$ ) and frequently observed ghost boutons in the $d n r x(2.188 \pm 0.430, N=16)$ (Figures 8B$\left.\mathbf{B}^{\prime \prime}\right)$, dnlg1 $(0.750 \pm 0.250, N=8)$ (Figures $\left.8 \mathbf{C}-\mathbf{C}^{\prime \prime}\right)$, dnlg2 $(0.625 \pm 0.154, N=16)$ (Figures 8D- $\left.\mathbf{D}^{\prime \prime}\right)$, dnlg3 $(1.00 \pm 0.327$, $N=8)$ (Figures $\left.8 \mathrm{E}-\mathbf{E}^{\prime \prime}\right)$, and $\operatorname{dnlg} 4(1.00 \pm 0.224, N=16)$ 


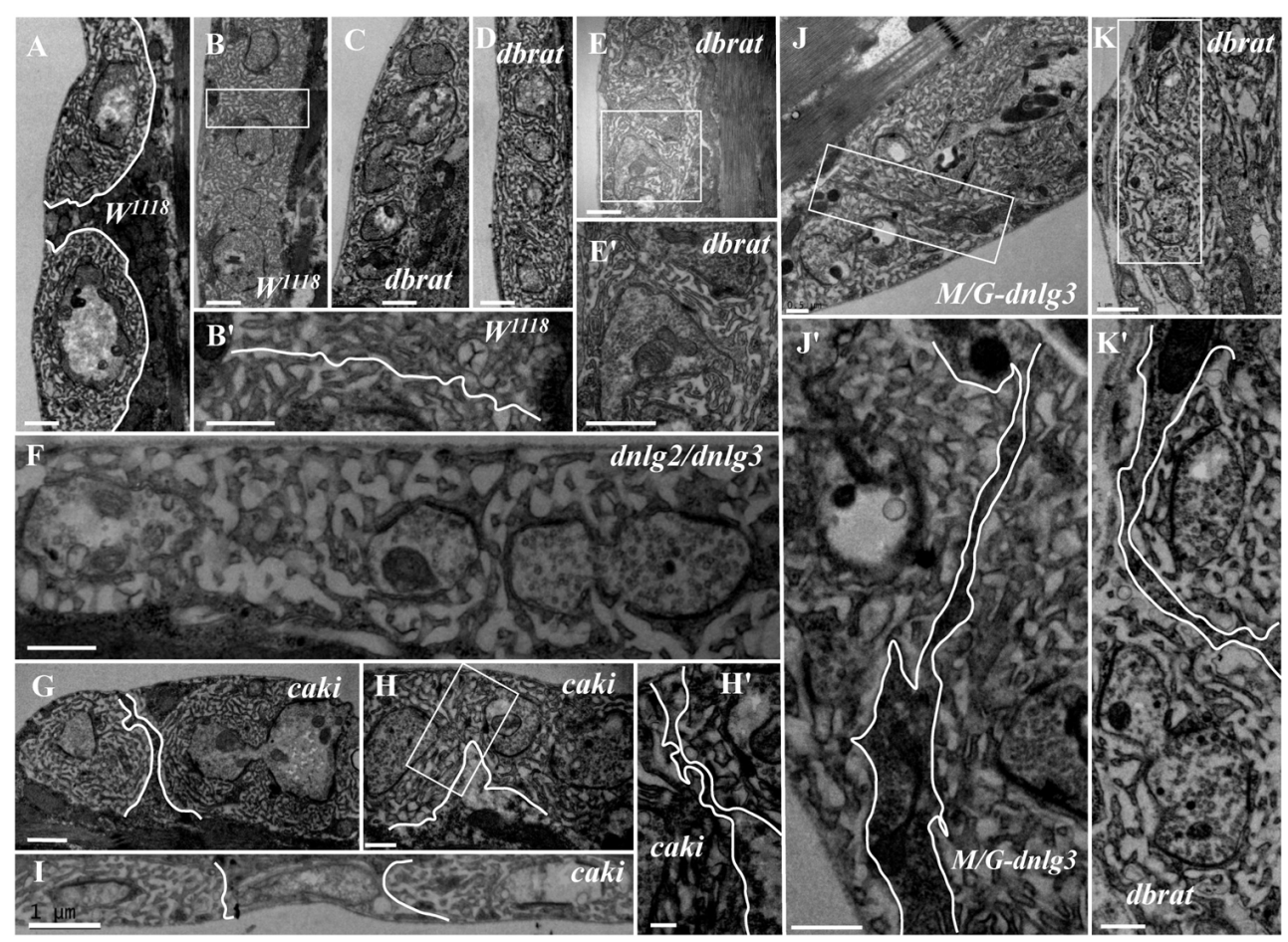

FIGURE 6 | Ultrastructural comparison of small boutons and atypical satellite boutons. Both adjacent type lb boutons in the wild-type strain have independent SSR membranes (A-B'). The atypical satellite boutons contain several beaded small boutons, and they are wrapped in the same SSR membrane in the dbrat mutant (C-E') and in the dnlg2; dnlg3 double mutant (F). The small beaded boutons have obvious boundaries and orientations between the small-sized boutons in the caki

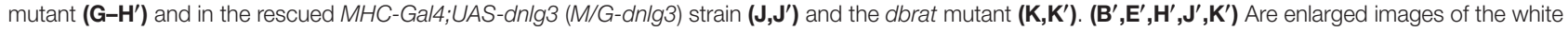

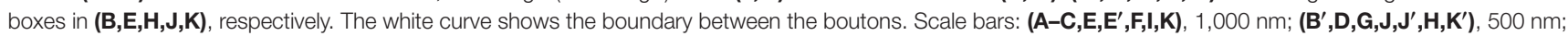
$\left(\mathbf{H}^{\prime}\right), 200 \mathrm{~nm}$.

(Figures 8F-F $\mathbf{F}^{\prime \prime}$ ) mutants (Figure 9G and Table 2). The $d n r x$ mutant had the most ghost boutons, exhibiting significantly more than that the $d n \lg 1(P=0.035)$ and $d n \lg 2(P=0.0018)$ mutants (Figure 9G). The number of ghost boutons was not significantly different among the four $d n \lg$ mutants (Figure 9G).

No satellite boutons were observed in the wild-type strain or in the $d n r x, d n \lg 1, d n \lg 2, d n \lg 3$, and $d n \lg 4$ mutants (data not shown), but satellite boutons were frequently observed in all overexpression strains of $d n r x(1.000 \pm 0.204$, $N=16)$ (Figures 9A, $\left.\mathbf{A}^{\prime}\right), \operatorname{dn} \lg 1(1.583 \pm 0.193, N=12)$ (Figures 9B, $\left.\mathbf{B}^{\prime}\right), \operatorname{dnlg} 2(0.625 \pm 0.263, N=8)$ (Figures 9C, $\mathbf{C}^{\prime}$ ), dnlg3 (1.125 $\pm 0.227, N=8$ ) (Figures 9D, $\mathbf{D}^{\prime}$ ), and $d n \lg 4$ (1.125 $\pm 0.350, N=8$ ) (Figures 9E, $\mathbf{E}^{\prime}, 9 \mathrm{H}$ and Table 2). More satellite boutons were observed in the 24B-Gal4;UAS- $d n l g 3$ strain (data not shown) than in the MHC-Gal4;UAS-dnlg3 strain (Figures 9D, $\mathbf{D}^{\prime}$ ), but the difference between the two strains was not significant. Consistent with the electron microscopy results, satellite boutons (3.667 $\pm 0.432, N=12)$ frequently appeared in the $d n \lg 2 ; d n \lg 3$ double mutant (Figures $\mathbf{9 F}, \mathbf{F}^{\prime}, \mathbf{G}$ and Table 2), and up to six small boutons surrounded the main bouton (Figures 9F, $\mathbf{F}^{\prime}$ ).

Interestingly, ghost boutons frequently appeared in the $d n \lg 2 ; \operatorname{dnlg} 3$ double mutant $(1.750 \pm 0.392, N=12)\left(\right.$ Figure $\left.9 F^{\prime \prime}\right)$ and in the strains overexpressing $d n r x(1.000 \pm 0.275, N=12)$ (Figure 9A' $\left.\mathbf{A}^{\prime \prime}\right), d n l g 1(0.625 \pm 0.193, N=8)\left(\right.$ Figure 9B $\left.{ }^{\prime \prime}\right), d n l g 2$
$(0.416 \pm 0.102, N=24)$ (Figure $\left.9 C^{\prime \prime}\right), d n l g 3(0.625 \pm 0.263$, $N=8)$ (Figure 9D' ${ }^{\prime \prime}$, and dnlg4 (0.667 $\left.\pm 0.156, N=24\right)$ (Figure $9 \mathbf{E}^{\prime \prime}$ ). The number of satellite boutons in the $d n \lg 2 ; d n \lg 3$ double mutant was significantly higher than that in the other overexpression strains (Figure 9H), and the number of ghost boutons was significantly higher than those of the five overexpression strains (Figure 9G).

These findings indicated that appropriate doses of $d n r x$ and $d n l g s$ maintained the normal development of the synapse. The $d n r x$ and $4 d n \lg$ mutations led to ghost boutons that were markers of poor synaptic development, and the overexpression of $d n r x$ and the four $d n \operatorname{lgs}$ led to satellite boutons that were markers of excessive synaptic development. Excessive synaptic development could also lead to ghost boutons.

\section{DISCUSSION}

\section{The Characteristics and Growth of Ghost Boutons on TEM}

Ghost boutons are undifferentiated and immature synaptic boutons that contain synaptic vesicles and lack active zones and postsynaptic structures, although these structures can differentiate into mature boutons over prolonged periods (Ataman et al., 2008) and do exist in wild-type organisms at 


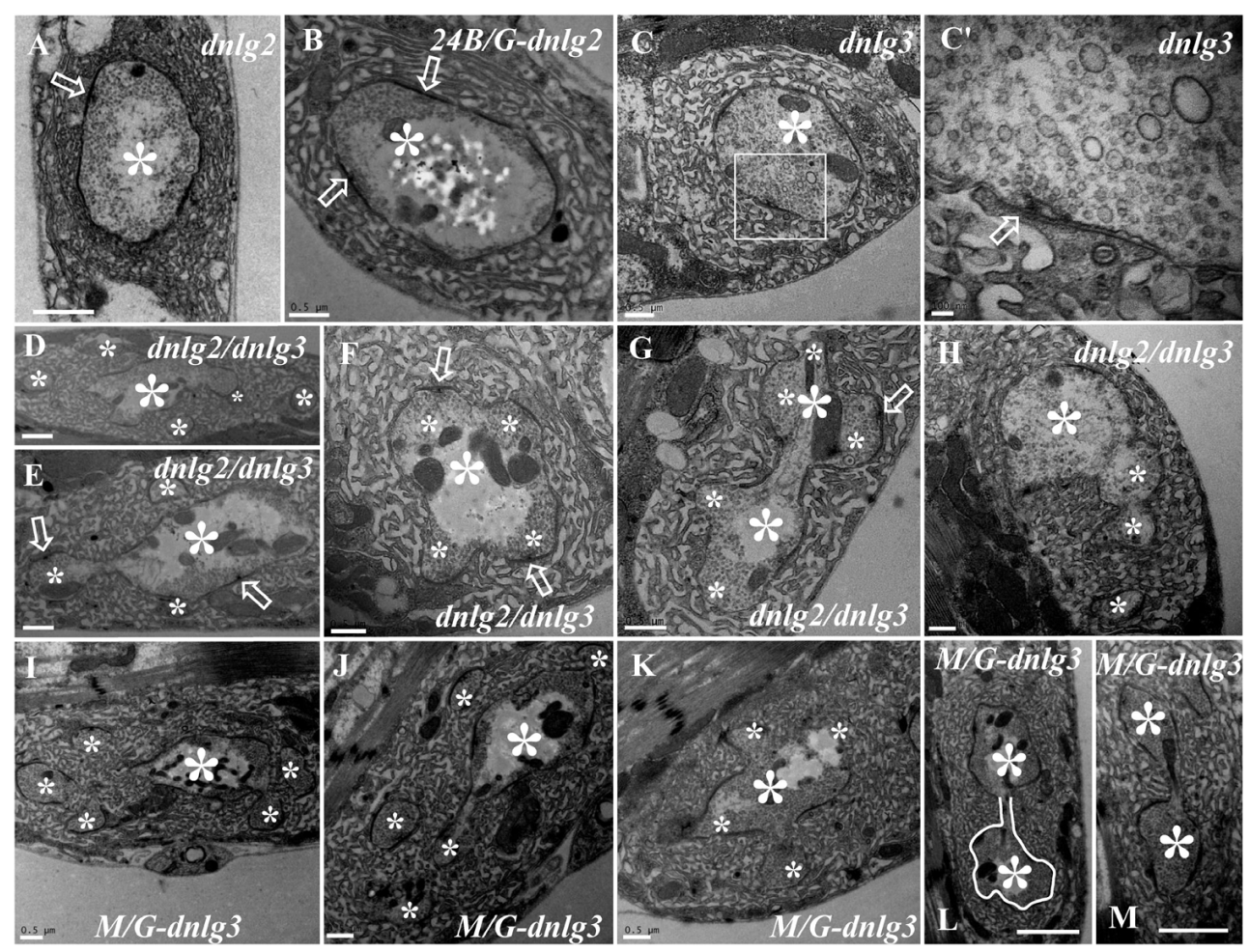

FIGURE 7 | DNIg2 and DNIg3 synergistically regulate satellite boutons on transmission electron microscopy (TEM). No satellite boutons in the dnlg2 mutant (A), the rescued 24B-Gal4;UAS-dnlg2 strain (B), and the dnlg3 (C, $\left.\mathbf{C}^{\prime}\right)$ mutant. The satellite boutons in the dnlg2; dnlg3 double mutant (D-E). The cruciform bouton (F), exogenous small boutons (G), and repeatedly growing small boutons from a large neuromuscular junction (NMJ) bouton (H). Satellite boutons around the main bouton in the rescued MHC-Ga/4;UAS-dnlg3 strain (I,J). More cruciform boutons (K). The large irregular boutons in a common SSR membrane (L,M). Hollow arrows show T-bars and synapses, large asterisks show the main boutons, and small asterisks show small boutons or branches. The white curve shows an irregular bouton shape. Scale bars: (A-C,D-K), $500 \mathrm{~nm}$; (C'), $100 \mathrm{~nm}$; (L,M), 1,000 nm.

a very low frequency, as observed with confocal microscopy (Ataman et al., 2006; Loya et al., 2014) but not with TEM. Ghost bouton budding was observable from both type Ib and type Is boutons (Piccioli and Littleton, 2014) under confocal microscopy. Ghost boutons are regulated by bone morphogenetic protein (BMP) (Piccioli and Littleton, 2014), Wnt signaling molecules (Packard et al., 2002; Ataman et al., 2008), and PFN1 mutants (Wu et al., 2017).

Ultrastructural evidence also confirmed that ghost boutons were poorly developed in the NMJ bouton. Under TEM, ghost boutons lacked active zones, SSR membranes, and mitochondria and had dense clear synaptic vesicles (Packard et al., 2002). Furthermore, we found that ghost boutons contained synaptic vesicles with multiple diameters in wild-type flies and mutant strains; the large synaptic vesicles were similar to those in the immature axonal neurites of the Drosophila larval ventral nerve cord (Gan et al., 2014), while the large vesicles almost completely disappeared in both NMJ type Ib boutons (Jia et al., 1993) and mature axonal neurites (Gan et al., 2014). Ghost boutons contained very few signals for Brp (Ataman et al., 2006, 2008), a marker of synaptic active zones and T-bar structures. Early NMJ type Ib boutons in Drosophila embryos had frequent T-bar structures and mitochondria but no SSR membrane (Prokop et al., 1996; Koper et al., 2012).
We observed few T-bar structures in the ghost boutons in the $d n r x$ mutant, for which the presynaptic structure could be independently formed without the induction of a postsynaptic structure (Prokop et al., 1996). Furthermore, ghost boutons could be differentiated into mature boutons with a dense SSR over prolonged periods (Ataman et al., 2008), and we observed sparse linear SSR membranes that developed the SSR in wild-type flies and in the $d n r x, d n \lg 2$, and dnlg4 mutants.

The SSR membrane originated from the muscle cell membrane, stretched to the ghost bouton, and enfolded and branched into the axon terminal. We observed clear and obvious sites in wild-type (Figures $\mathbf{4} \mathbf{A}^{\prime}, \mathbf{B}^{\prime}$ ) and $d n r x$ (Figure $3 \mathbf{D}^{\prime \prime}$ ) and dnlg1 mutant (Figures $4 \mathbf{D}, \mathbf{D}^{\prime}$ ) flies, directly indicating that the SSR membranes of the NMJ bouton originated from different sites of the muscle cell membrane and developed into complicated SSR membranes from multiple sources. Furthermore, the invaginated sites formed different layers of the SSR membranes; the outer layer of the SSR membrane from the outer invaginated sites formed the outer layers of the SSR membrane in the dnlg1 mutant (Figures $4 \mathbf{C}-\mathbf{D}^{\prime}$ ), while the innermost invaginated sites formed synapses. Then, the single SSR membrane was repeatedly branched (Figures $4 \mathbf{A}^{\prime \prime}, \mathbf{B}^{\prime \prime}$ ) and formed the complex SSR of the NMJ bouton. 


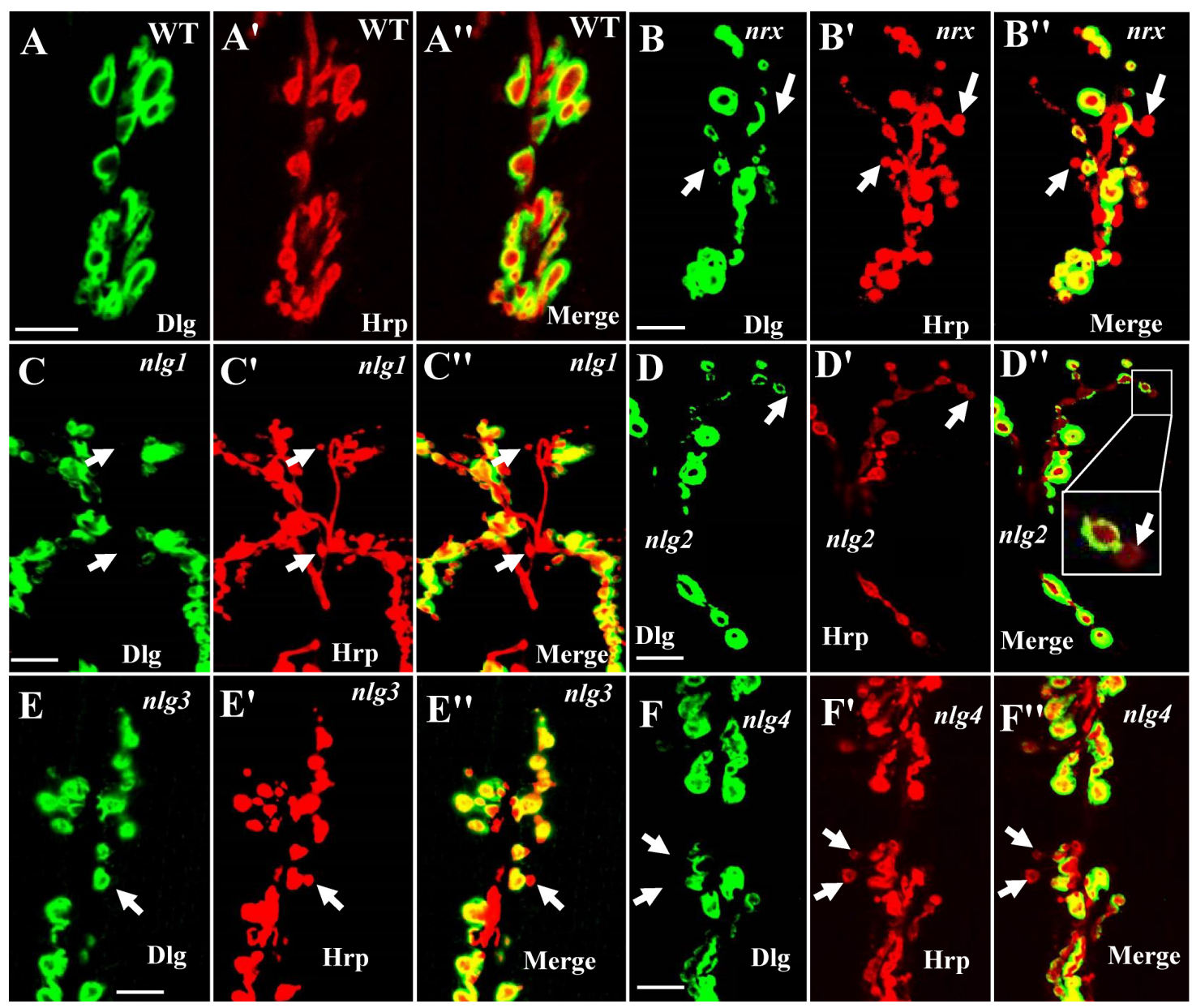

FIGURE 8 | Ghost boutons in the $d n r x$ mutant and 4 dnlg mutants. Almost no ghost boutons were found in wild-type flies (A-A'). Ghost boutons in the $d n r x$

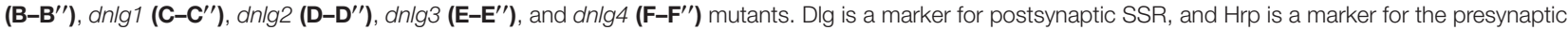
membrane. The thick white arrows show the ghost boutons that have no Dlg signals. Scale bars, $10 \mu \mathrm{m}$.

The developing ghost bouton had a relatively independent SSR membrane. In the dnrx and dnlg4 mutants, the sparse SSR membranes stretched toward and touched the ghost boutons from different directions, and these membranes could not generate the SSR membrane of the other bouton. Furthermore, the ghost bouton might have been adjacent to a type Is bouton, but there was a clear boundary between the ghost bouton and the type Is bouton (Figure $\mathbf{3} \mathbf{B}^{\prime \prime}$ ) in the dnlg4 mutant. Moreover, the ghost boutons contained synapse and T-bar structures (Figure 2A) with sparse SSR membranes in the other directions (Figures 4D-D' ${ }^{\prime \prime}$ ).

\section{Mutations in dnrx and dnlgs Lead to Ghost Boutons}

The SSR membrane was swollen near the ghost boutons (Figure $\mathbf{1 \mathbf { E } ^ { \prime }}$ ) in wild-type flies and was thin in the $d n r x$ (Figure $2 \mathbf{B}^{\prime}$ ), $d n \lg 2$ (Figure $2 \mathbf{C}^{\prime \prime}$ ), and $d n \lg 4$ (Figures $3 \mathbf{D}^{\prime}, \mathbf{D}^{\prime \prime}$ ) mutants, which frequently showed ghost boutons. Therefore, the swollen SSR membrane had components from the muscle cell membrane, which could contribute to normal SSR membrane branching and development; a reduction in or the absence of the swollen SSR membrane could result in ghost boutons that did not have SSR membranes or with thin SSR membranes in type Ib boutons in the dnlg2 mutant (Sun et al., 2011) or with a small SSR area in type Ib boutons in the dnlg4 mutant (Li et al., 2013; Zhang et al., 2017). In fact, the SSR thickness was reduced in the dnrx mutant (data not shown).

Neurexins and neuroligins are synaptic adhesion molecules commonly associated with autism and schizophrenia (Zhang et al., 2018) and are reportedly involved in synapse formation and synaptic transmission in Drosophila NMJ type Ib boutons (Sun et al., 2009, 2011). Although we prepared serial slices, we did not observe typical ghost boutons in the dnlg1 and $d n \lg 3$ mutants by TEM, possibly due to occlusion of the grids. Therefore, we analyzed the ghost bouton numbers by confocal microscopy with a large field and three-dimensional view. We confirmed that ghost boutons were regulated by the five synaptic adhesion molecules, DNrx, DNlg1, DNlg2, DNlg3, and DNlg4, in this study. Interestingly, all the five related mutants had defects in 


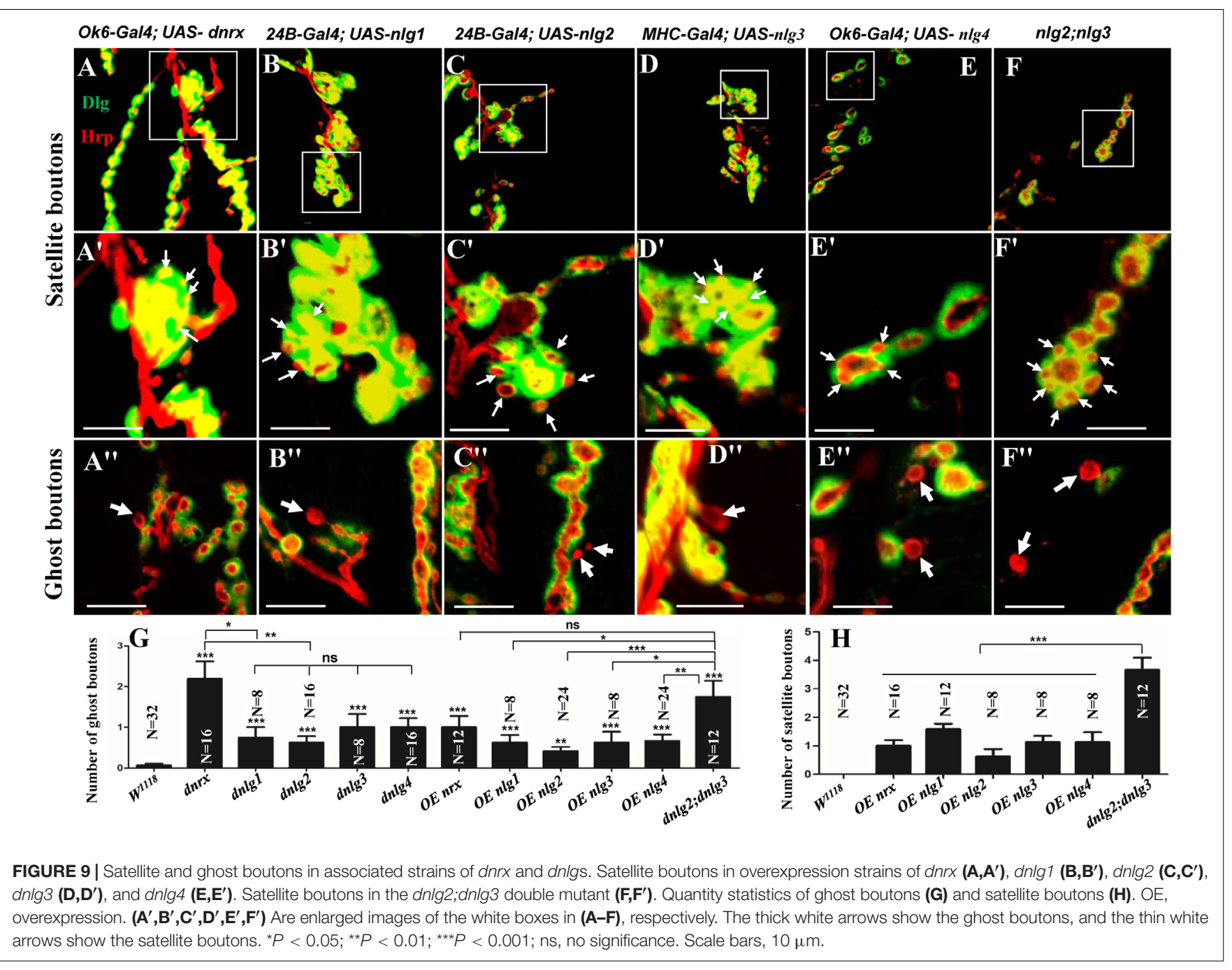

synaptic morphology and function in NMJ, which could lead to ghost boutons, the poorly developed boutons.

Ghost boutons are also regulated by the BMP (Piccioli and Littleton, 2014) and Wnt signaling molecules (Ataman et al., 2008; Packard et al., 2002) and by PFN1 (Wu et al., 2017), and these molecules were involved in regulation of the cytoskeletal network. DNrx, DNlg1, and DNlg4 regulated synaptic growth via the BMP signaling pathway at the Drosophila NMJ (Banerjee et al., 2017; Zhang et al., 2017), and DNrx, DNlg1, DNlg2, and DNlg3 regulated postsynaptic actin filament (Xing et al., 2018), a key cytoskeletal component in NMJ, which demonstrated that DNrx and DNlgs mediated synaptic cytoskeleton disorder and caused ghost boutons.

Although DNrx was generally distributed in the presynaptic membrane, DNlgs were distributed in the postsynaptic membrane, and mutations in $d n r x, d n \lg 1, d n \lg 2, d n \lg 3$, and dnlg4 led to the frequent occurrence of ghost boutons, which suggested that poorly developed boutons or bouton-related genetic changes might be associated with autism. Therefore, with respect to autism caused by DNrx and DNlgs, attention should be paid to not only their associations with impaired synaptic transmission but also their induction of ghost boutons, the immature synaptic boutons.

\section{The Characteristics of Satellite Boutons on TEM}

Satellite boutons are described as parent boutons of normal sizes with many attached small boutons (Menon et al., 2013), and these structures bud from axonal segments connecting two adjacent boutons on TEM (Torroja et al., 1999). In this study, we characterized three subtypes of satellite boutons with TEM. The typical satellite bouton comprises a large main bouton and several small boutons, with the main bouton in the center and the small boutons, appearing as several satellites, budding around the main bouton. If the main bouton or parent bouton was also small, only a few small boutons were grouped together, which could be considered an atypical satellite bouton with a shared SSR membrane (Figures 7E,F). Based on confocal microscopy, the small NMJ boutons were shown to be distributed as beads in both the caki (Sun et al., 2009) and dbrat (Shi et al., 2013) mutants, and distinguishing between the small-sized boutons and 


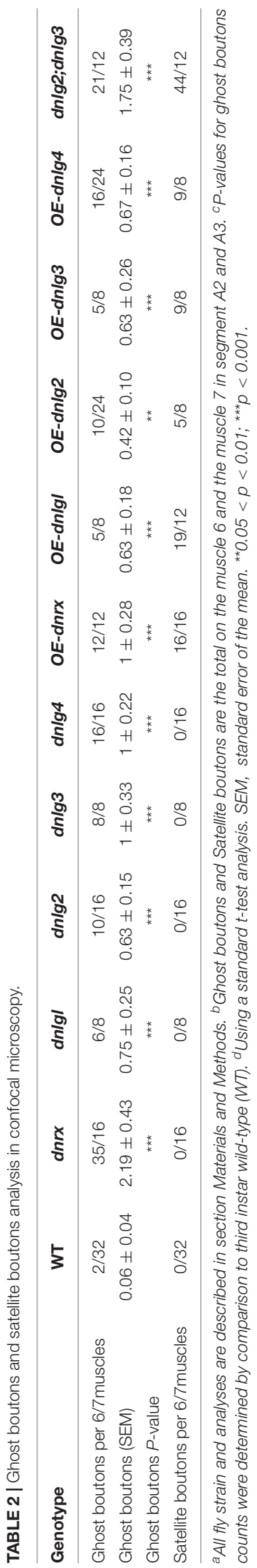

small satellite boutons was difficult. In TEM, the small, beaded NMJ boutons shared SSR membranes in the dbrat mutant and the $d n \lg 2 ; \operatorname{dnlg} 3$ mutant (Figure 6), and we believed that the small boutons represented the other atypical satellite bouton variety. In the caki, dbrat, and dnlg2;dnlg3 mutants, the small beaded boutons were simple abnormally small boutons with obvious boundaries and orientations (Figure 6). Therefore, the shared SSR membrane among the NMJ boutons was used to identify satellite boutons on TEM.

\section{Overexpressing DNrx and DNIgs Leads to Satellite Boutons}

In contrast to the mutations of $d n r x$ and dnlgs leading to ghost boutons, satellite boutons were observed in all five strains overexpressing $d n r x$ and dnlgs. The satellite boutons were promoted by $\mathrm{DNlg} 4$ overexpression and required BMP signaling (Zhang et al., 2017). Since DNrx and DNlg1 also regulated synaptic growth via the BMP signaling pathway (Banerjee et al., 2017), the satellite boutons in the strains of DNrx and DNlg1 overexpression were likely to be regulated via the BMP signaling pathway. No satellite boutons were observed in the rescued $24 B-$ Gal4;UAS-nlg2 strain, but satellite boutons frequently appeared in the 24B-Gal4;UAS-nlg2 overexpression strain, demonstrating that an appropriate dose of DNlg2 regulated the overgrowth of synaptic boutons. Moreover, DNlg4 also had a dosage-sensitive genetic interaction with the components of the BMP pathway (Zhang et al., 2017). No satellite boutons were observed in the $d n r x, d n \lg 1, d n \lg 2$, dnlg3, and $d n \lg 4$ mutants. However, satellite boutons frequently appeared in the $d n \lg 2 ; d n \lg 3$ double mutants, which suggested that the postsynaptic adhesion molecules $d n \lg 2$ and $d n l g 3$ synergistically regulated satellite boutons that could be considered an indicator of synaptic overgrowth.

\section{DNrx and DNIgs Regulate the Balance of Ghost and Satellite Boutons}

Neurexin and neuroligins are well-known synaptic adhesion molecules associated with autism, and they have close evolutionary homologs in invertebrates and vertebrates. There are three $n r x$ genes in mammalians but only one neurexin gene in Drosphila. There are four nlg genes in rodents, which encode $\mathrm{Nlg} 1, \mathrm{Nlg} 2$, Nlg3, and Nlg4, while five $n l g$ genes, nlg1, nlg2, nlg3, $n \lg 4$, and $n \lg 4 y$, in the human genome. The $n \lg 4 y$ was similar in structure to that which encodes Nlg4Y on the Y chromosome. However, the four $d n l g$ genes, encoding DNlg1-4, were not one-to-one homologous to mammalian Nlg1-4, and they were similar to human Nlg1 in homology, which showed that the four $d n l g$ genes were fairly convergent on specific phenotypes, such as satellite boutons and ghost bouton.

All mutants of $d n r x$ and dnlgs had abnormal presynaptic and postsynaptic phenotypes. $d n r x$ and $d n l g 4$ mainly played presynaptic roles (Li et al., 2007; Zhang et al., 2017), dnlg1 and dnlg3 mainly played postsynaptic functions (Banovic et al., 2010; Xing et al., 2018), and $d n l g 2$ played pre- and postsynaptic roles (Sun et al., 2011; Chen et al., 2012). Therefore, $d n r x$ and dnlgs regulated the presynaptic and postsynaptic cytoskeletal networks through their extracellular domains and jointly maintained the 


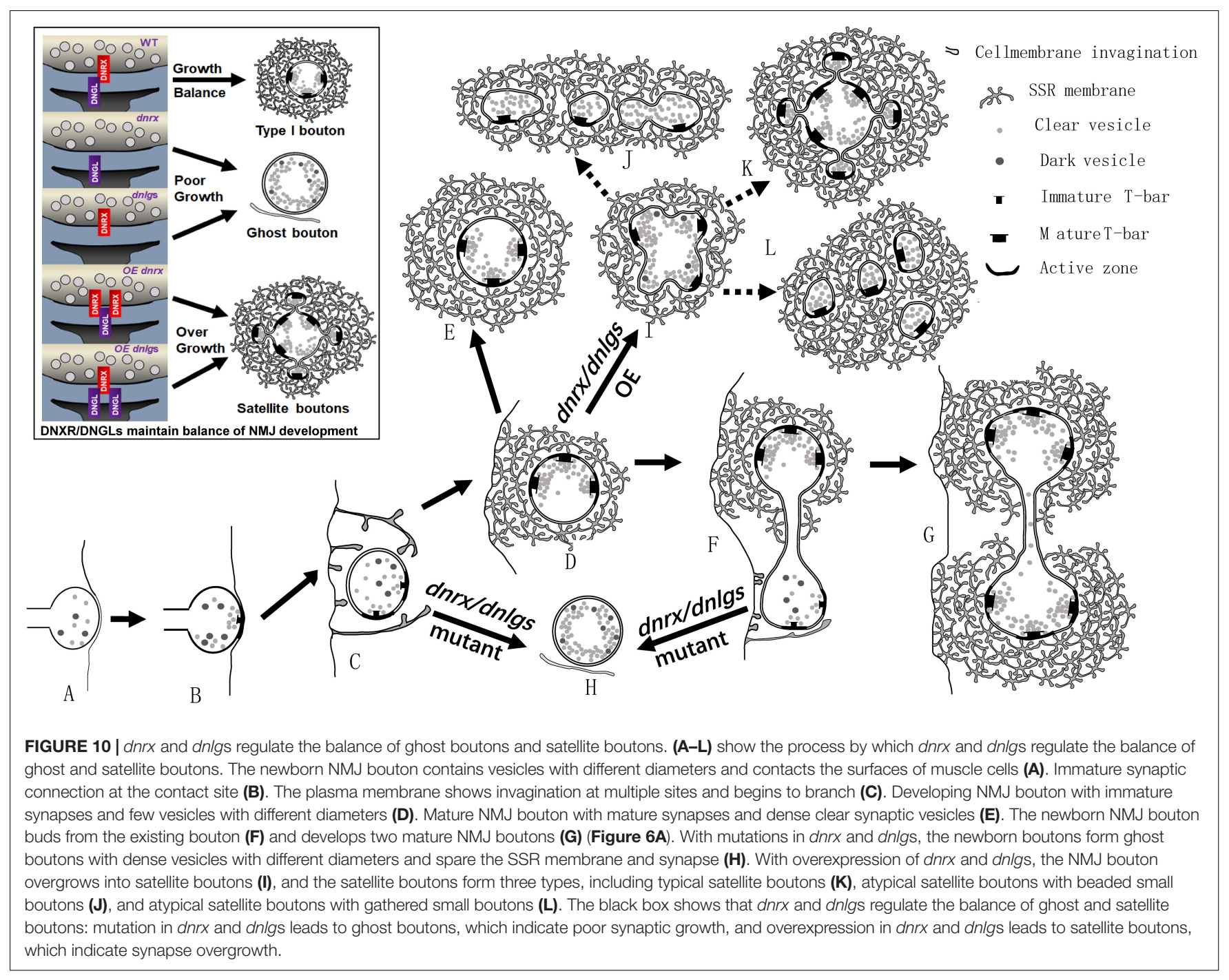

development of NMJ boutons in Drosophila. For a single NMJ bouton, dnrx, dnlg1, dnlg2, dnlg3, and dnlg4 mutations led to ghost boutons that indicate poor development in the NMJ bouton. The overexpression of $d n r x$ and the four $d n l g s$ and the dnlg2/dnlg 3 double mutation led to satellite boutons that could indicate overgrowth. These findings suggested that both DNrx and DNlgs jointly maintained the balance of poor development and overgrowth in NMJ boutons by regulating ghost and satellite boutons (Figure 10 black box).

Based on the above data, we described the pattern of ghost and satellite boutons. The vacuolar axon terminal with vesicles of different diameters reached and contacted the surfaces of muscle cells (Figure 10A; Koper et al., 2012; Gan et al., 2014), and the vesicles gradually underwent synaptic vesicle docking and immature T-bar assembly (Figure 10B; Koper et al., 2012; Gan et al., 2014). The axon terminal invaginated into the muscle cell and formed double-layer membranes with the cell membrane (Figure 10C). Then, the other plasma membrane invaginated and enfolded the axon terminal from multiple sites (Figure 10D), and the SSR membrane further branched and folded (Jia et al., 1993), finally developing into mature NMJ boutons with mature T-bar structures and dense synaptic vesicles (Figure 10E; Jia et al., 1993; Gan et al., 2014). The newborn bouton without an SSR was derived (Figure 10F) from the existing NMJ bouton during development (Zito et al., 1999) and developed into another mature NMJ bouton (Figure 10G). Once the developing NMJ bouton (Figure 10C) or the newborn bouton (Figure 10F) was disturbed, ghost boutons with vesicles of different diameters formed and spared the SSR membrane and synapse (Figure 10H). The number of ghost boutons was regulated by DNrx and the four DNlgs as well as by DNT1, DNT2, Spz (Sutcliffe et al., 2013), LIM kinase (Piccioli and Littleton, 2014), PFN1 (Wu et al., 2017), and physicochemical factors (Ataman et al., 2008).

If the NMJ boutons developed with a common SSR membrane, they would have budded like yeast (Figure 10I; Lee and $\mathrm{Wu}, 2010)$ and developed into mature satellite boutons (Lee and $\mathrm{Wu}, 2010$ ). The mature satellite boutons had a typical appearance with small surrounding boutons (Figure 10K) and two atypical morphologies with small beaded boutons (Figure 10J) and small gathered boutons (Figure 10L). 


\section{DATA AVAILABILITY STATEMENT}

All datasets generated for this study are included in the article/supplementary material.

\section{AUTHOR CONTRIBUTIONS}

All authors had full access to all data in the study and take responsibility for the integrity of the data and the accuracy of the data analysis. GG and XW: study concept and design. GG, GJ, and MY: acquisition of the data, analysis and interpretation of the data. GG and ZC: drafting of the manuscript, critical revision of the manuscript for important intellectual content, administrative, technical, and material support.

\section{REFERENCES}

Ataman, B., Ashley, J., Gorczyca, M., Ramachandran, P., Fouquet, W., Sigrist, S. J., et al. (2008). Rapid activity-dependent modifications in synaptic structure and function require bidirectional Wnt signaling. Neuron 57, 705-718. doi: 10.1016/j.neuron.2008.01.026

Ataman, B., Budnik, V., and Thomas, U. (2006). Scaffolding proteins at the Drosophila neuromuscular junction. Int. Rev. Neurobiol. 75, 181-216. doi: 10.1016/S0074-7742(06)75009-7

Atwood, H. L., Govind, C. K., and Wu, C. F. (1993). Differential ultrastructure of synaptic terminals on ventral longitudinal abdominal muscles in Drosophila larvae. J. Neurobiol. 24, 1008-1024. doi: 10.1002/neu.480240803

Banerjee, S., Venkatesan, A., and Bhat, M. A. (2017). Neurexin, Neuroligin and Wishful Thinking coordinate synaptic cytoarchitecture and growth at neuromuscular junctions. Mol. Cell Neurosci. 78, 9-24. doi: 10.1016/j.mcn.2016. 11.004

Banovic, D., Khorramshahi, O., Owald, D., Wichmann, C., Riedt, T., Fouquet, W., et al. (2010). Drosophila neuroligin 1 promotes growth and postsynaptic differentiation at glutamatergic neuromuscular junctions. Neuron 66, 724-738. doi: 10.1016/j.neuron.2010.05.020

Chai, A., Withers, J., Koh, Y. H., Parry, K., Bao, H., Zhang, B., et al. (2008). hVAPB, the causative gene of a heterogeneous group of motor neuron diseases in humans, is functionally interchangeable with its Drosophila homologue DVAP-33A at the neuromuscular junction. Hum. Mol. Genet. 17, 266-280. doi: $10.1093 / \mathrm{hmg} / \mathrm{ddm} 303$

Chen, Y. C., Lin, Y. Q., Banerjee, S., Venken, K., Li, J., Ismat, A., et al. (2012). Drosophila neuroligin 2 is required presynaptically and postsynaptically for proper synaptic differentiation and synaptic transmission. J. Neurosci. 32, 16018-16030. doi: 10.1523/JNEUROSCI.1685-12.2012

Dickman, D. K., Lu, Z., Meinertzhagen, I. A., and Schwarz, T. L. (2006). Altered synaptic development and active zone spacing in endocytosis mutants. Curr. Biol. 16, 591-598. doi: 10.1016/j.cub.2006.02.058

Endris, V., Wogatzky, B., Leimer, U., Bartsch, D., Zatyka, M., Latif, F., et al. (2002). The novel Rho-GTPase activating gene MEGAP/ srGAP3 has a putative role in severe mental retardation. Proc. Natl. Acad. Sci. U.S.A. 99, 11754-11759. doi: 10.1073/pnas.162241099

Fuentes-Medel, Y., Logan, M. A., Ashley, J., Ataman, B., Budnik, V., and Freeman, M. R. (2009). Glia and muscle sculpt neuromuscular arbors by engulfing destabilized synaptic boutons and shed presynaptic debris. PLoS Biol. 7:e1000184. doi: 10.1371/journal.pbio.1000184

Gan, G., Lv, H., and Xie, W. (2014). Morphological identification and development of neurite in Drosophila ventral nerve cord neuropil. PLoS One 9:e105497. doi: 10.1371/journal.pone.0105497

Gan, G., and Zhang, C. (2018). The precise subcellular localization of Dlg in the Drosophila larva body wall using improved pre-embedding immuno-EM. J. Neurosci. Res. 96, 467-480. doi: 10.1002/jnr.24139
GG and MY: statistical analysis. GG: funding and study supervision.

\section{FUNDING}

This work was supported by the Southeast University Fundamental Research Fund (3224005416).

\section{ACKNOWLEDGMENTS}

We are indebted to Dr. Zhang Yongqing for gifting us the $d b r a t^{11}$ and dbrat $^{192}$ strains. We are grateful for the Electron Microscopy Laboratory, School of Medicine, Southeast University, Nanjing, which provided all the data.

Jia, X. X., Gorczyca, M., and Budnik, V. (1993). Ultrastructure of neuromuscular junctions in Drosophila: comparison of wild type and mutants with increased excitability. J. Neurobiol. 24, 1025-1044.

Koper, A., Schenck, A., and Prokop, A. (2012). Analysis of adhesion molecules and basement membrane contributions to synaptic adhesion at the Drosophila embryonic NMJ. PLoS One 7:e36339. doi: 10.1371/journal.pone.003 6339

Larkin, A., Chen, M. Y., Kirszenblat, L., Reinhard, J., van Swinderen, B., and Claudianos, C. (2015). Neurexin-1 regulates sleep and synaptic plasticity in Drosophila melanogaster. Eur. J. Neurosci. 42, 2455-2466. doi: 10.1111/ejn. 13023

Lee, J., and Wu, C. F. (2010). Orchestration of stepwise synaptic growth by K+ and Ca2+ channels in Drosophila. J. Neurosci. 30, 15821-15833. doi: 10.1523/ JNEUROSCI.3448-10.2010

Li, J., Ashley, J., Budnik, V., and Bhat, M. A. (2007). Crucial role of Drosophila neurexin in proper active zone apposition to postsynaptic densities, synaptic growth, and synaptic transmission. Neuron 55, 741-755.

Li, W., Yao, A., Zhi, H., Kaur, K., Zhu, Y. C., Jia, M., et al. (2016). Angelman syndrome protein Ube3a regulates synaptic growth and endocytosis by inhibiting BMP signaling in Drosophila. PLoS Genet. 12:e1006062. doi: 10.1371/ journal.pgen.1006062

Li, Y., Zhou, Z., Zhang, X., Tong, H., Li, P., Zhang, Z. C., et al. (2013). Drosophila neuroligin 4 regulates sleep through modulating GABA transmission. J. Neurosci. 33, 15545-15554. doi: 10.1523/JNEUROSCI.0819-13.2013

Loya, C. M., McNeill, E. M., Bao, H., Zhang, B., and Van Vactor, D. (2014). miR- 8 controls synapse structure by repression of the actin regulator enabled. Development 141, 1864-1874. doi: 10.1242/dev.105791

Menon, K. P., Carrillo, R. A., and Zinn, K. (2013). Development and plasticity of the Drosophila larval neuromuscular junction. Wiley Interdiscip. Rev. Dev. Biol. 2, 647-670. doi: 10.1002/wdev.108

Miller, D. L., Ballard, S. L., and Ganetzky, B. (2012). Analysis of synaptic growth and function in Drosophila with an extended larval stage. J. Neurosci. 32, 13776-13786. doi: 10.1523/JNEUROSCI.0508-12.2012

Monastirioti, M., Gorczyca, M., Rapus, J., Eckert, M., White, K., and Budnik, V. (1995). Octopamine immunoreactivity in the fruit fly Drosophila melanogaster. J. Comp. Neurol. 356, 275-287.

Packard, M., Koo, E. S., Gorczyca, M., Sharpe, J., Cumberledge, S., and Budnik, V. (2002). The Drosophila Wnt, wingless, provides an essential signal for pre- and postsynaptic differentiation. Cell 111, 319-330.

Piccioli, Z. D., and Littleton, J. T. (2014). Retrograde BMP signaling modulates rapid activity-dependent synaptic growth via presynaptic LIM kinase regulation of cofilin. J. Neurosci. 34, 4371-4381. doi: 10.1523/JNEUROSCI.4943-13. 2014

Prokop, A., Landgraf, M., Rushton, E., Broadie, K., and Bate, M. (1996). Presynaptic development at the Drosophila neuromuscular junction: assembly and localization of presynaptic active zones. Neuron 17, 617-626. 
Rui, M., Qian, J., Liu, L., Cai, Y., Lv, H., Han, J., et al. (2017). The neuronal protein Neurexin directly interacts with the Scribble-Pix complex to stimulate F-actin assembly for synaptic vesicle clustering. J. Biol. Chem. 292, 14334-14348. doi: 10.1074/jbc.M117.794040

Sen, A., Yokokura, T., Kankel, M. W., Dimlich, D. N., Manent, J., Sanyal, S., et al. (2011). Modeling spinal muscular atrophy in Drosophila links Smn to FGF signaling. J. Cell Biol. 192, 481-495. doi: 10.1083/jcb.201004016

Shi, W., Chen, Y., Gan, G., Wang, D., Ren, J., Wang, Q., et al. (2013). Brain tumor regulates neuromuscular synapse growth and endocytosis in Drosophila by suppressing mad expression. J. Neurosci. 33, 12352-12363. doi: 10.1523/ JNEUROSCI.0386-13.2013

Sun, M., Liu, L., Zeng, X., Xu, M., Liu, L., Fang, M., et al. (2009). Genetic interaction between Neurexin and CAKI/CMG is important for synaptic function in Drosophila neuromuscular junction. Neurosci. Res. 64, 362-371. doi: 10.1016/j. neures.2009.04.009

Sun, M., Xing, G., Yuan, L., Gan, G., Knight, D., With, S. I., et al. (2011). Neuroligin 2 is required for synapse development and function at the Drosophila neuromuscular junction. J. Neurosci. 31, 687-699. doi: 10.1523/JNEUROSCI. 3854-10.2011

Sutcliffe, B., Forero, M. G., Zhu, B., Robinson, I. M., and Hidalgo, A. (2013). Neuron-type specific functions of DNT1, DNT2 and Spz at the Drosophila neuromuscular junction. PLoS One 8:e75902. doi: 10.1371/journal.pone. 0075902

Tong, H., Li, Q., Zhang, Z. C., Li, Y., and Han, J. (2016). Neurexin regulates nighttime sleep by modulating synaptic transmission. Sci. Rep. 6:38246. doi: $10.1038 /$ srep38246

Torroja, L., Packard, M., Gorczyca, M., White, K., and Budnik, V. (1999). The Drosophila beta-amyloid precursor protein homolog promotes synapse differentiation at the neuromuscular junction. J. Neurosci. 19, 7793-7803.

Tsuda, H., Han, S. M., Yang, Y., Tong, C., Lin, Y. Q., Mohan, K., et al. (2008). The amyotrophic lateral sclerosis 8 protein VAPB is cleaved, secreted, and acts as a ligand for Eph receptors. Cell 133, 963-977. doi: 10.1016/j.cell.2008. 04.039

Tsuda, H., Jafar-Nejad, H., Patel, A. J., Sun, Y., Chen, H. K., Rose, M. F., et al. (2005). The AXH domain of Ataxin-1 mediates neurodegeneration through its interaction with Gfi-1/Senseless proteins. Cell 122, 633-644.
Wu, C. H., Giampetruzzi, A., Tran, H., Fallini, C., Gao, F. B., and Landers, J. E. (2017). A Drosophila model of ALS reveals a partial loss of function of causative human PFN1 mutants. Hum. Mol. Genet. 26, 2146-2155. doi: 10.1093/hmg/ ddx 112

Xing, G., Gan, G., Chen, D., Sun, M., Yi, J., Lv, H., et al. (2014). Drosophila neuroligin3 regulates neuromuscular junction development and synaptic differentiation. J. Biol. Chem. 289, 31867-31877. doi: 10.1074/jbc.M114.574897

Xing, G., Li, M., Sun, Y., Rui, M., Zhuang, Y., Lv, H., et al. (2018). NeurexinNeuroligin 1 regulates synaptic morphology and functions via the WAVE regulatory complex in Drosophila neuromuscular junction. eLife 7:e30457. doi: 10.7554/eLife.30457

Zempel, H., and Mandelkow, E. M. (2015). Tau missorting and spastininduced microtubule disruption in neurodegeneration: Alzheimer disease and hereditary spastic paraplegia. Mol. Neurodegener. 10:68. doi: 10.1186/s13024015-0064-1

Zhang, P., Lu, H., Peixoto, R. T., Pines, M. K., Ge, Y., Oku, S., et al. (2018). Heparan sulfate organizes neuronal synapses through neurexin partnerships. Cell 174:1450-1464.e23. doi: 10.1016/j.cell.2018.07.002

Zhang, X., Rui, M., Gan, G., Huang, C., Yi, J., Lv, H., et al. (2017). Neuroligin 4 regulates synaptic growth via the bone morphogenetic protein (BMP) signaling pathway at the Drosophila neuromuscular junction. J. Biol. Chem. 292, 1799118005. doi: 10.1074/jbc.M117.810242

Zito, K., Parnas, D., Fetter, R. D., Isacoff, E. Y., and Goodman, C. S. (1999). Watching a synapse grow: noninvasive confocal imaging of synaptic growth in Drosophila. Neuron 22, 719-729.

Conflict of Interest: The authors declare that the research was conducted in the absence of any commercial or financial relationships that could be construed as a potential conflict of interest.

Copyright (c) 2020 Guangming, Junhua, Chenchen, Yang and Wei. This is an openaccess article distributed under the terms of the Creative Commons Attribution License (CC BY). The use, distribution or reproduction in other forums is permitted, provided the original author(s) and the copyright owner(s) are credited and that the original publication in this journal is cited, in accordance with accepted academic practice. No use, distribution or reproduction is permitted which does not comply with these terms. 